\title{
Reactive Loading of Arbitrarily Illuminated Cylinders to Minimize Microwave Backscatter
}

\author{
Kun-Mu Chen ${ }^{2}$ \\ Department of Electrical Engineering, Michigan State University, East Lansing, Mich.
}

(September 23, 1964)

\begin{abstract}
In this paper a study of the minimization of the radar cross section of a thin cylinder by central loading is presented. The induced current on a center-loaded cylinder illuminated by a plane wave at an arbitrary angle is determined. The backscattered field is calculated and the optimum loading to achieve zero broadside backscattering is obtained. An optimum impedance loaded at the center of a resonant cylinder can reduce its radar cross section more than $30 \mathrm{~dB}$ for any aspect angle. For an antiresonant cylinder an optimum central loading can minimize the radar cross section in the broadside direction but it cannot modify appreciably the radar cross section in the off-broadside direction. The effectiveness and the feasibility of the loading technique for the reduction of the radar cross section of a metallic body is discussed.
\end{abstract}

\section{Introduction}

Many investigations have been made of methods of reducing the radar cross section of metallic bodies, especially with regard to applications to radar camouflage techniques. Two methods have been widely used: the first utilizes radar-absorbing materials, the second consists of reshaping the body to change the reflection pattern. A third method, known as the method of reactive loading, is the subject of investigation of this paper.

The first known use of reactive loading to minimize the backscattering cross section was made by Iams [1950] who applied the technique to metallic posts in a parallel-plate pillbox structure. Shortly after this, Sletten [1962] employed the method to decrease the radar cross section of objects in space. Several other authors [King, 1956; Hu, 1958; Ås and Schmitt, 1958] have also studied the cross section of a cylinder with and without a central load. These investigations indicated that the cross section of a half-wavelength cylinder can be significantly reduced by the use of a high reactive impedance load at its center.

Chen and Liepa [1964a] reported on the use of reactive loading to reduce the broadside backscattering from cylinders illuminated at normal incidence. They showed how an optimum loading could be found for zero broadside backscattering. In the present paper, the study is generalized to the case of arbitrary incidence and considers the case of backscattering in an arbitrary direction. An article on doubly loaded cylinders is in preparation.

The problem is studied by considering the current induced in a body illuminated by an electromagnetic wave. We consider the case of a plane wave which illuminates a perfectly conducting cylinder whose radius is small and whose length is less than two wavelengths. The plane wave induces a current on the cylinder; this in turn produces a scattered electromagnetic field. If an impedance is added at the center of the cylinder, the induced current is modified; hence, so is the scattered field. An optimum loading of the cylinder will reduce the magnitude of the induced current and reverse its phase over some part of the cylinder. As the result the scattered field can be minimized in a direction over an aspect range.

\footnotetext{
${ }^{1}$ The research in this paper was supported by Air Force Cambridge Research Laboratories under Contract AF 19(628)-2374

${ }^{2}$ Formerly with the University of Michigan, Ann Arbor, Mich.
} 
For a center-loaded cylinder the induced current is first determined as a function of the cylinder dimensions, the midpoint impedance, and the incident electric field. Using this solution we calculate the scattered field and obtain an optimum impedance, i.e., an impedance which gives a minimum backscattering. In order to verify this solution, the calculated values of the radar cross sections of loaded cylinders are compared with the experimental results by Chen and Liepa [1964b]. Excellent agreement is found between theory and experiment.

Throughout the study a resonant cylinder whose total length is equal to $0.43 \lambda$ ( $\lambda=$ wavelength) and an antiresonant cylinder of total length $0.85 \lambda$ or $0.9 \lambda$ are used as typical examples. In the interest of simplicity, the analysis is limited to the case of a thin cylinder. The length of the cylinder is assumed to be shorter than two wavelengths. This dimension is in the so-called resonance region and is of special importance in radar detection. MKS rationalized units are used in the analysis and the time-dependence factor $e^{i \omega t}$ is omitted.

\section{Induced Current on a Center-Loaded Cylinder Illuminated by a Plane Wave at an Arbitrary Angle}

When a plane electromagnetic wave is obliquely incident on a center-loaded cylinder the induced current can be divided into a symmetrical and an antisymmetrical component. The symmetrical component is predominant in a resonant cylinder while in an antiresonant cylinder the antisymmetrical component dominates. A midpoint impedance has a strong effect on the symmetrical component of the induced current but does not affect the antisymmetrical component. For this reason central loading is very effective in reducing the backscattering in the broadside direction but cannot modify appreciably that in the off-broadside direction.

As the first step of the analysis, the induced current on a center-loaded cylinder illuminated by a plane wave at an arbitrary angle is determined as a function of the cylinder dimensions, the midpoint impedance, and the incident electric field. The integral equation method is used. It is worthwhile to note that King [1956] solved a similar problem of a center-loaded receiving antenna but ignored the antisymmetrical component of the antenna current. His results are also too complicated for our purpose. We use a somewhat different method to determine the induced current on a center-loaded cylinder as an explicit function of the midpoint impedance.

\subsection{Integral Equation for the Induced Current}

The geometry of the problem is as shown in figure 1. A cylinder with a radius $a$ and length $2 h$ is assumed to be perfectly conducting. A plane EM wave is incident to the cylinder at an angle

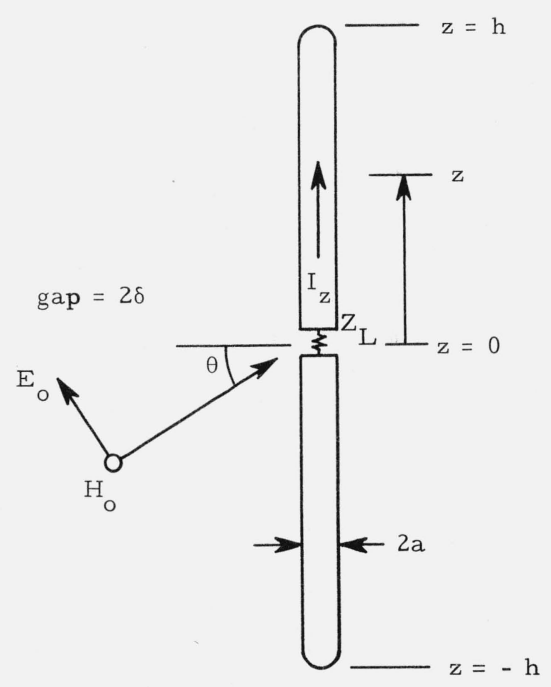

FigURE 1. A center-loaded cylinder illuminated obliquely by a plane EM wave. 
$\theta$. A lumped impedance $Z_{L}$ is connected at the center of the cylinder. The dimensions of interest are

$$
\begin{aligned}
& \frac{1}{4} \lambda<2 h<2 \lambda \\
& \beta_{0}^{2} a^{2}<<1,
\end{aligned}
$$

where $\lambda$ is the wavelength and $\beta_{0}$ is the wave number. The second condition implies that the cylinder is thin and only the axial current is induced.

The tangential component of the incident $E$ field along the cylinder is assumed to be

$$
E_{z}^{i n}=E_{0} \cos \theta e^{-j \beta_{0}} \sin \theta z
$$

where $E_{0}$ is a constant.

The current and the charge on the cylinder maintain a tangential electrical field at the surface which can be expressed as

$$
E_{z}^{a}=-\frac{\partial \phi}{\partial z}-j \omega A_{z}
$$

where $\phi$ is the scalar potential maintained by the charge and $A_{z}$ is the tangential component of the vector potential maintained by the current. By using the Lorentz condition,

$$
\phi=j \frac{\omega}{\beta_{0}^{2}} \nabla \cdot \vec{A}
$$

Equation (2) can be expressed as

$$
E_{z}^{a}=-j \frac{\omega}{\beta_{0}^{2}}\left(\frac{\partial^{2}}{\partial z^{2}}+\beta_{0}^{2}\right) A_{z}
$$

The electric field maintained across the gap at the center of the cylinder can be expressed as

$$
E_{z}^{g}=Z_{L} I_{0} \delta(z)
$$

where $Z_{L}$ is the center load, $I_{0}$ is the induced current at the center of the cylinder and $\delta(z)$ is a delta function.

Since the tangential electric field should be continuous at the boundary we obtain the following equation

$$
E_{z}^{a}+E_{z}^{i n}=Z_{L} I_{0} \delta(z)
$$

$$
\text { for }-h<z<h \text {. }
$$

Equation (6) implies that the total tangential electric field vanishes on the surface of the cylinder and maintains a voltage drop of $Z_{L} I_{0}$ across the gap at the center of the cylinder.

The substitution of (1) and (4) in (6) gives

$$
\begin{gathered}
\frac{\partial^{2}}{\partial z^{2}} A_{z}+\beta_{0}^{2} A_{z}=-j \frac{\beta_{0}^{2}}{\omega}\left[E_{0} \cos \theta e^{-j \beta_{0} \sin \theta z}-Z_{L} I_{0} \delta(z)\right] \\
\text { for }-h<z<h .
\end{gathered}
$$


The general solution for $A_{z}$ is the sum of the complementary function and a particular integral

$$
A_{z}=\frac{-j}{v_{0}}\left[C_{1} \cos \beta_{0} z+C_{2} \sin \beta_{0} z+\Theta(z)\right],
$$

where $v_{0}$ is $1 / \sqrt{\mu_{0} \epsilon_{0}}, C_{1}$ and $C_{2}$ are arbitrary constants, and where the particular integral $\Theta(z)$ can be obtained as

$$
\Theta(z)=\frac{E_{0}}{\beta_{0} \cos \theta} e^{-j \beta_{0} \sin \theta z}-\frac{1}{2} Z_{L^{\prime}} I_{0} \sin \beta_{0}|z|
$$

In (8), $A_{z}$ can be divided into a symmetrical and an antisymmetrical component

$$
A_{z}(z)=A_{z}^{s}(z)+A_{z}^{a}(z)
$$

Thus

$$
\begin{aligned}
& A_{z}^{s}(z)=\frac{-j}{v_{0}}\left[C_{1} \cos \beta_{0} z+\frac{E_{0}}{\beta_{0} \cos \theta} \cos \left(\beta_{0} z \sin \theta\right)-\frac{1}{2} Z_{L} I_{0} \sin \beta_{0}|z|\right] \\
& A_{z}^{a}(z)=\frac{-j}{v_{0}}\left[C_{2} \sin \beta_{0} z-j \frac{E_{0}}{\beta_{0} \cos \theta} \sin \left(\beta_{0} z \sin \theta\right)\right] .
\end{aligned}
$$

We also divide the induced current on the cylinder into a symmetrical and an antisymmetrical component:

$$
I_{z}(z)=I_{z}^{s}(z)+I_{z}^{a}(z)
$$

and, by the assumed symmetries,

$$
I_{z}^{s}(z)=I_{z}^{s}(-z), \quad I_{z}^{a}(z)=-I_{z}^{a}(-z)
$$

From the definition of the vector potential, we can write $A_{z}$ in terms of $I_{z}$ as follows:

$$
\begin{array}{r}
A_{z}^{s}(z)=\frac{\mu_{0}}{4 \pi} \int_{-h}^{h} I_{z}^{s}\left(z^{\prime}\right) K_{a}\left(z, z^{\prime}\right) d z^{\prime}=\frac{-j}{v_{0}}\left[C_{1} \cos \beta_{0} z+\frac{E_{0}}{\beta_{0} \cos \theta} \cos \left(\beta_{0} z \sin \theta\right)-\frac{1}{2} Z_{L} I_{0} \sin \beta_{0}|z|\right] \\
A_{z}^{a}(z)=\frac{\mu_{0}}{4 \pi} \int_{-h}^{h} I_{z}^{a}\left(z^{\prime}\right) K_{a}\left(z, z^{\prime}\right) d z^{\prime}=\frac{-j}{v_{0}}\left[C_{2} \sin \beta_{0} z-j \frac{E_{0}}{\beta_{0} \cos \theta} \sin \left(\beta_{0} z \sin \theta\right)\right]
\end{array}
$$

where

$$
K_{a}\left(z, z^{\prime}\right)=\frac{e^{-j \beta_{0} \sqrt{\left(z-z^{\prime}\right)^{2}+a^{2}}}}{\sqrt{\left(z-z^{\prime}\right)^{2}+a^{2}}}
$$

Equations (14) and (15) are integral equations for the induced currents, $I_{z}^{s}(z)$ and $I_{z}^{a}(z)$.

\subsection{Symmetrical Component of the Induced Current}

The symmetrical component of the induced current $I_{z}^{s}(z)$ is determined from (14). The details are given in appendix 1 . The final solution for $I_{z}^{s}(z)$ is 


$$
\begin{array}{r}
I_{z}^{s}(z)=\frac{j E_{0}}{30 \beta_{0} \cos \theta}\left\{\left[\frac{\cos \left(\beta_{0} h \sin \theta\right)-M_{2} T_{\theta}-N_{2} T_{s a}}{\cos \beta_{0} h-M_{1} T_{c a}-N_{1} T_{s a}}\right] M_{1}\left(\cos \beta_{0} z-\cos \beta_{0} h\right)\right. \\
+\left[\frac{N_{1} \cos \left(\beta_{0} h \sin \theta\right)-N_{1} M_{2} T_{\theta}+M_{1} N_{2} T_{c a}-N_{2} \cos \beta_{0} h}{\cos \beta_{0} h-M_{1} T_{c a}-N_{1} T_{s a}}\right] \sin \beta_{0}(h-|z|) \\
\left.\quad-M_{2}\left[\cos \left(\beta_{0} z \sin \theta\right)-\cos \left(\beta_{0} h \sin \theta\right)\right]\right\}
\end{array}
$$

where

$$
\begin{gathered}
M_{1}=\frac{1}{T_{c d}}\left(1-\cos \beta_{0} h\right) \\
N_{1}=\frac{-Z_{L} \sin \beta_{0} h\left(1-\cos \beta_{0} h\right)^{2}}{T_{c d} Z_{L} \sin ^{2} \beta_{0} h-j 60 T_{c d} T_{s d} \cos \beta_{0} h} \\
M_{2}=\frac{1}{T_{\theta d}}\left[1-\cos \left(\beta_{0} h \sin \theta\right)\right] \\
N_{2}=\frac{-Z_{L} \sin \beta_{0} h\left[1-\cos \left(\beta_{0} h \sin \theta\right)\right]^{2}}{T_{\theta d} Z_{L} \sin ^{2} \beta_{0} h-j 60 T_{\theta d} T_{s d} \cos \beta_{0} h} .
\end{gathered}
$$

The constants, $T_{c d}, T_{s d}, T_{\theta d}, T_{c a}, T_{s a}$, and $T_{\theta a}$ are defined in appendix 1 . They are functions of cylinder size, $h$ and $a$, and the angle, $\theta$, of the incident EM wave.

Equation (17) gives a complete expression for the symmetrical component of the induced current on a cylinder with a center load $Z_{L}$, when illuminated obliquely by a plane EM wave at an angle $\theta ; I_{z}^{s}(z)$ is expressed as a function of the cylinder size, the center load $Z_{L}$, and the incident angle $\theta$ of an EM wave.

\subsection{Antisymmetrical Component of the Induced Current}

By solving (15), the antisymmetrical component of the induced current $I_{z}^{a}(z)$ can be determined as in appendix 2. The final solution for $I_{z}^{a}(z)$ is

$I_{z}^{a}(z)=\frac{E_{0}}{30 \beta_{0} \cos \theta}\left[\frac{\frac{1}{2} \sin \left(\beta_{0} h \sin \theta\right) \sec \frac{\beta_{0} h}{2}-\sin \left(\frac{\beta_{0} h}{2} \sin \theta\right)}{T_{a}(h / 2)-\frac{1}{2} \sec \beta_{0} h T_{a}(h)}\right]$

$$
\cdot\left[\sin \left(\beta_{0} h \sin \theta\right) \sin \beta_{0} z-\sin \beta_{0} h \sin \left(\beta_{0} z \sin \theta\right)\right] \cdot
$$

The constants $T_{a}(h / 2)$ and $T_{a}(h)$ are defined in appendix 2 as functions of cylinder size, $h$ and $a$, and the angle, $\theta$, of the incident EM wave.

Equation (22) gives the complete solution for the antisymmetrical component of the induced current on a cylinder with a center load $Z_{L}$ and illuminated obliquely by a plane EM wave at an angle $\theta$. The important point we observe from $(22)$ is that $I_{z}^{a}(z)$ is a function of the cylinder size and the incident angle only, and is entirely independent of the center load $Z_{L}$. The result is not surprising because $I_{z}^{a}(z)$ is always zero at the center of the cylinder and an impedance loaded at the center of the cylinder cannot have any effect on $I_{z}^{a}(z)$.

The total current induced on a cylinder is the sum of $I_{z}^{s}(z)$ and $I_{z}^{a}(z)$. Since $I_{z}^{s}(z)$ is a strong function of $Z_{L}$ and $I_{z}^{a}(z)$ is independent of $Z_{L}$, the introduction of an impedance at the center of the cylinder can modify $I_{z}^{s}(z)$ greatly but does not affect $I_{z}^{a}(z)$. In general, $I_{z}^{a}(z)$ is zero or small compared with $I_{z}^{s}(z)$ when $\theta$ is zero or small. Therefore, we can expect to minimize the backscattering at or near the broadside direction by central loading because the backscattering in these directions is due mainly to $I_{z}^{s}(z)$. Moreover, in a resonant cylinder $(2 h \sim(1 / 2+n) \lambda), I_{z}^{s}(z)$ is the 
predominant component and central loading can actually reduce the backscattering in any aspect angle. In an antiresonant cylinder $(2 h \sim n \lambda)$, central loading can minimize the broadside backscattering but the off-broadside backscattering at $\theta \simeq 40^{\circ}$ cannot be reduced by central loading because it is due to a large $I_{z}^{a}(z)$. .

Some examples in the following section will illustrate these phenomena more clearly.

\section{Numerical Examples for Some Special Cases}

To show the dependence of the induced current on the center load $Z_{L}$, the cases of a resonant and an antiresonant cylinder illuminated by a plane wave at the broadside direction are considered in the first and the second example. The dependence of the induced current on the incident angle $\theta$ is shown in the third example.

\subsection{Induced Current on a Loaded Resonant Cylinder Illuminated by a Plane Wave at Normal Incidence}

When a plane wave is incident normally $\left(\theta=0^{\circ}\right)$ on a cylinder the backscattering is highest in this direction. The symmetrical component of the induced current $I_{z}^{s}(z)$ is maximum and the antisymmetrical component $I_{z}^{a}(z)$ is zero for this case. In a resonant cylinder with $2 h \simeq(1 / 2+n) \lambda$, we will see that the magnitude-and the phase of $I_{z}^{s}(z)$ can be significantly modified by a center load $Z_{L}$.

When $\theta=0^{\circ}, I_{z}^{s}(z)$ can be obtained from (17) to (21) as

$$
I_{z}^{s}(z)=\frac{j E_{0}}{30 \beta_{0}}\left[\frac{1}{\cos \beta_{0} h-M_{1} T_{c a}-N_{1} T_{s a}}\right]\left[M_{1}\left(\cos \beta_{0} z-\cos \beta_{0} h\right)+N_{1} \sin \beta_{0}(h-|z|)\right]
$$

where $M_{1}$ and $N_{1}$ are expressed in (18) and (19). It can be shown from (22) that $I_{z}^{a}(z)=0$ when $\theta=0^{\circ}$.

To present a numerical example, the specific case of

$$
a=0.0173 \lambda, \quad 2 h=0.43 \lambda, \quad Z_{L}=j X_{L}
$$

is considered. In this case only the purely reactive center loads are considered because they can be easily obtained, in practice, from a coaxial cavity which can be installed inside the cylinder.

The current distributions on the cylinder with various center loads are shown in figures 2 and 3. The strong dependence of $I_{z}^{s}(z)$ on $Z_{L}$ is evident in these two figures. The effect of $Z_{L}$ on $I_{z}^{s}(z)$ can be summarized as follows:

(1) When $Z_{L}=0$ the induced current is very large and distributes along the cylinder as a shifted cosine curve.

(2) When $Z_{L}=\infty$ the magnitude of the induced current is greatly reduced from the value for $Z_{L}=0$ and the distribution of the induced current becomes double humped with a null at the center.

(3) When $Z_{L}$ is capacitive and finite, the induced current is smaller than the case of $Z_{L}=0$ but larger than for $Z_{L}=\infty$.

(4) When $Z_{L}$ is inductive and finite, the magnitude of the induced current is smaller than the case of $Z_{L}=\infty$, and the induced current starts to have three loops along the cylinder, and it is of interest to note that the phase of the current at the center loop is reversed.

The most important and significant point is that when $Z_{L}$ is inductive and of some finite value, the magnitude of the induced current is reduced to a value even smaller than in the case of $Z_{L}$ $=\infty$ and the phase of the induced current is reversed at the center part of the cylinder. From this we can expect the existence of an optimum value for $Z_{L}$ which gives zero backscattering in the broadside direction. Actually this is the case as we can see in a later section.

The current distributions on this specific cylinder as functions of $Z_{L}$ shown in figures 2 and 3 have been confirmed experimentally by Chen and Liepa [1964A]. 


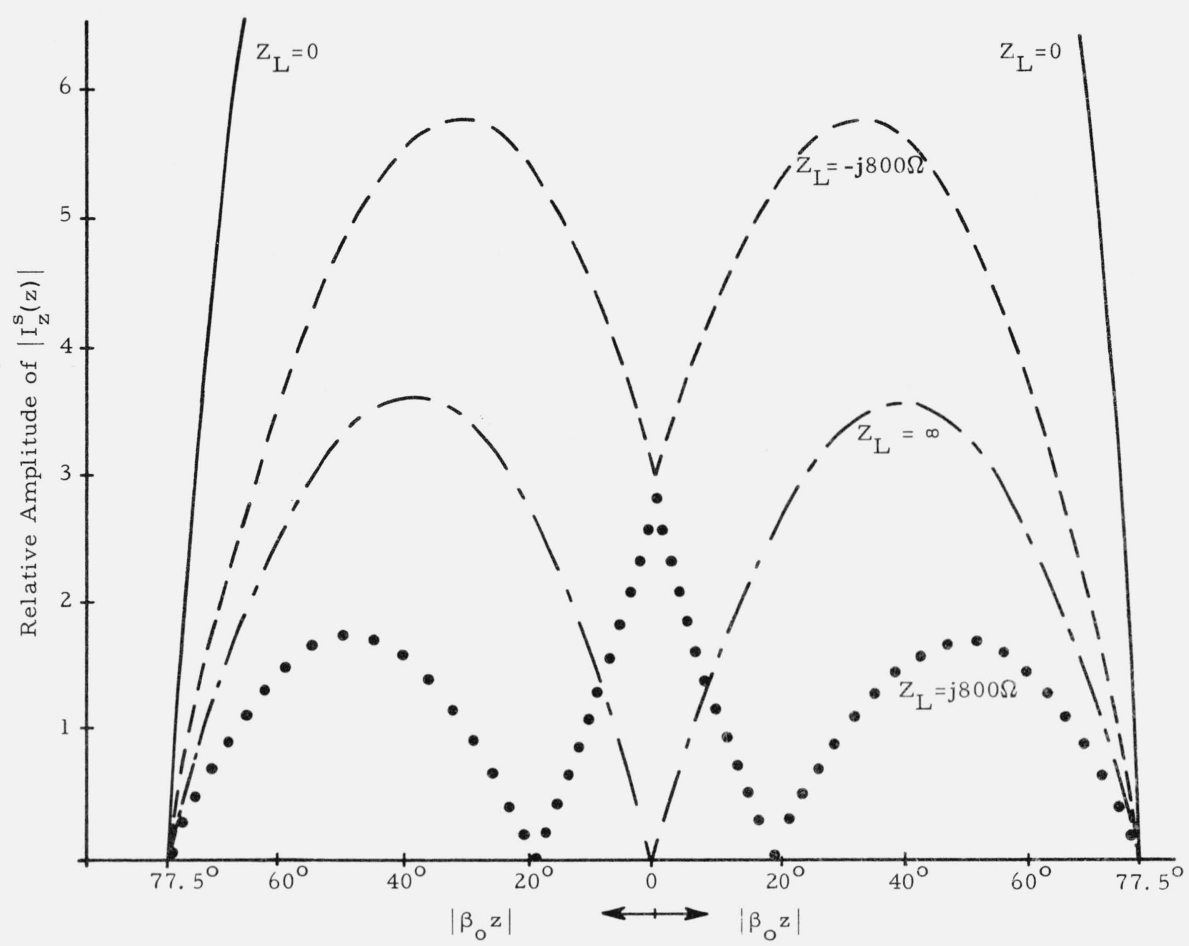

FIgURE 2. Current distribution, $\mathrm{I}_{\mathrm{z}}^{\mathrm{s}}(\mathrm{z})$, on cylinder of $\mathrm{h}=0.215 \lambda, \mathrm{a}=0.0173 \lambda, \theta=0^{\circ}$ for different central loads, $\mathrm{Z}_{\mathrm{L}}$.

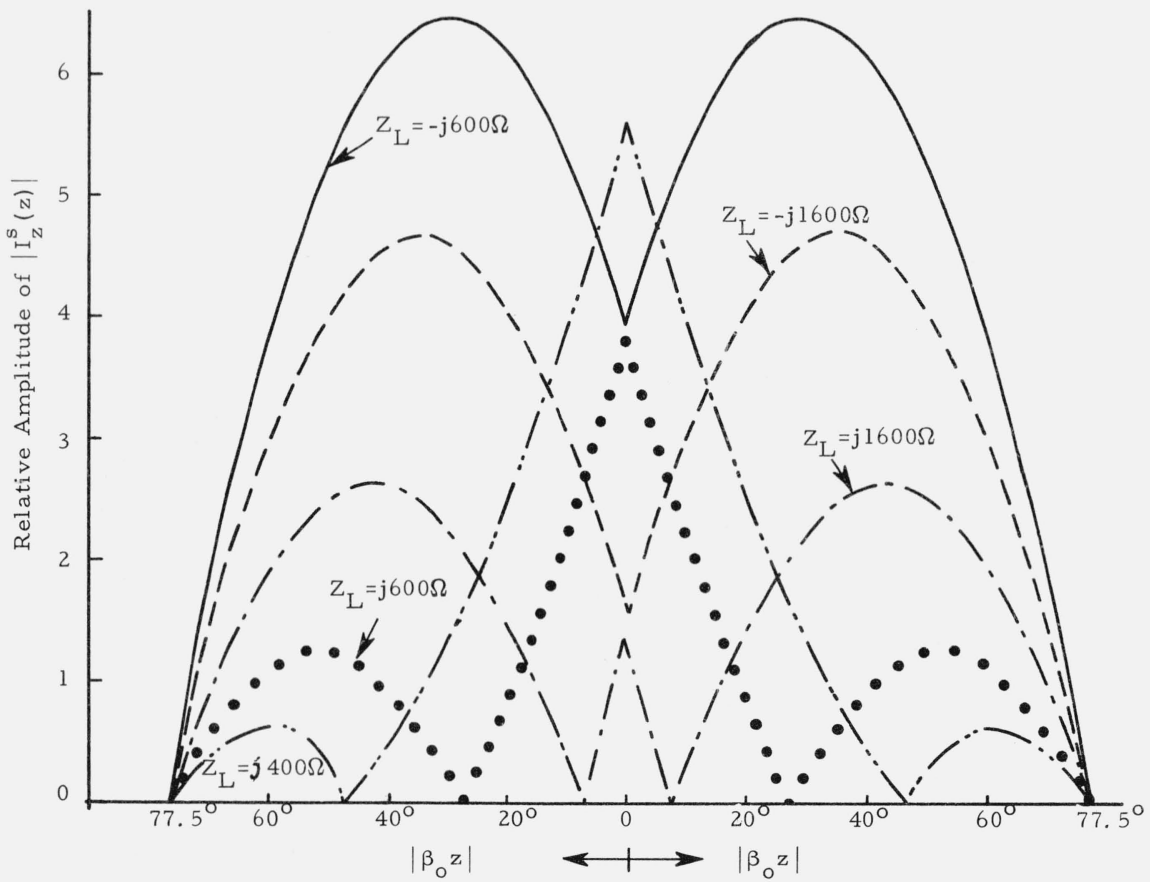

Figure 3. Current distribution, $\mathrm{I}_{z}^{\mathrm{s}}(\mathrm{z})$, on cylinder of $\mathrm{h}=0.215 \lambda, \mathrm{a}=0.0173 \lambda, \theta=0^{\circ}$ for different central loads, $\mathrm{Z}_{\mathrm{L}}$. 


\subsection{Induced Current on a Loaded Antiresonant Cylinder Illuminated by a Plane Wave at Normal Incidence}

An inductive impedance as the center load has been shown to be very effective in reducing the induced current on a resonant cylinder in the preceding example. In this example we will show that a purely reactive impedance is not very effective in reducing the induced current on an antiresonant cylinder with $2 h \simeq n \lambda$.

We choose a specific cylinder with antiresonant dimensions as

$$
a=0.0173 \lambda, \quad 2 h=0.9 \lambda, \quad Z_{L}=j X_{L} .
$$

The induced current is calculated from (23) for various $Z_{L}$. The current distributions on this cylinder with various $Z_{L}$ are shown graphically in figures 4 and 5 .

In these figures we observe that the introduction of a center load usually increases rather than decreases the induced current of an antiresonant cylinder. However, an inductive impedance tends to reverse the phase of the induced current at the center part of the cylinder as we see from the case of $Z_{L}=j 800 \Omega$ in figure 4 . This suggests that although a purely reactive impedance cannot reduce the backscattering of a cylinder to zero an inductive impedance with a resistive component may be used to reverse the phase of the induced current in such a way that the backscattering vanishes. (In effect, an optimum impedance for zero broadside backscattering from an antiresonant cylinder requires a large resistive component, as we will see in a later section.)

\subsection{Induced Current on a Loaded Cylinder as a Function of the Incident Angle of a Plane Wave}

In this example, the dependence of the induced current of a loaded cylinder on the incident angle of a plane wave is studied. We choose two typical cases, a resonant and an antiresonant cylinder both loaded with an impedance clo: - to the optimum value for zero broadside backscattering.

The first case is a resonant cylinder with $a=0.0173 \lambda, 2 h=0.43 \lambda$, and $Z_{L}=j 800 \Omega$. The distribution of the symmetrical component of the induced current, $I_{z}^{s}(z)$, for this $Z_{L}$ is about $20 \mathrm{~dB}$ lower than the value of $I_{z}^{s}(z)$ for $Z_{L}=0$ (see fig. 2). The information we obtained from figure 6 is that the distribution of $I_{z}^{s}(z)$ is essentially independent of incident angle except at the center of the cylinder. The antisymmetrical component of the induced current, $I_{z}^{a}(z)$, on this resonant cylinder is quite small as shown in figure 7. As already noted, $I_{z}^{a}(z)$ is entirely independent of $Z_{L}$ but it is strongly dependent on the cylinder size and on the incident angle. Thus $I_{z}^{a}(z)$ is zero when $\theta$ is zero and it reaches a maximum value when $\theta$ is about $45^{\circ}$. It is noted that the fact that the magnitudes of $I_{z}^{s}(z)$ and $I_{z}^{a}(z)$ are comparable in figures 6 and 7 is a consequence of the large reduction in $I_{z}^{s}(z)$ produced by the nearly optimum load $Z_{L}=j 800 \Omega$.

The second case is that of an antiresonant cylinder whose dimensions are $a=0.0173 \lambda$, $2 h=0.85 \lambda$, and $Z_{L}=j 600 \Omega$. The distributions of $I_{z}^{s}(z)$ for different incident angles are shown graphically in figure 8. Again we observe that the magnitude of $I_{z}^{s}(z)$ is only slightly affected by the incident angle. The antisymmetrical component of the induced current, $I_{z}^{a}(z)$, on this antiresonant cylinder is very large and its strong dependence upon the incident angle is shown in figure 9.

To summarize, we observe that while the antisymmetrical component of the induced current of a loaded cylinder is strongly dependent on the incident angle, the symmetrical component of the induced current is rather independent of the incident angle if the cylinder is loaded with an optimum (or near optimum) impedance. This phenomenon tends to indicate that an optimum central loading for zero broadside backscattering will remain effective for oblique incidence cases. Of course, a large antisymmetrical component of the current induced on an antiresonant cylinder for an oblique incidence can not be controlled by a center loading. This current can be modified only by the double-loading technique, which loads a cylinder at two points. The double-loading technique will be treated in a subsequent paper. 


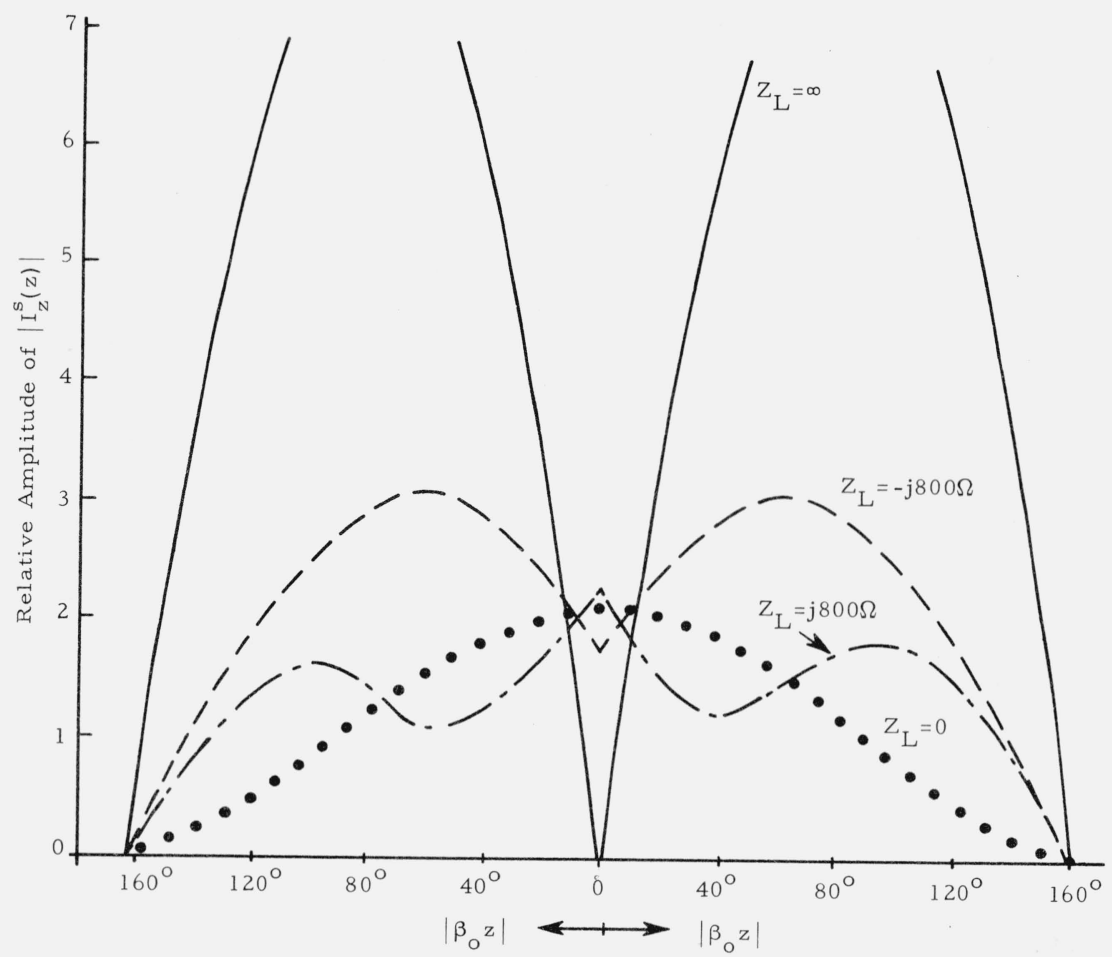

FIGURE 4. Current distribution, $\mathrm{I}_{\mathrm{z}}^{\mathrm{s}}(\mathrm{z})$, on cylinder of $\mathrm{h}=0.45 \lambda, \mathrm{a}=0.0173 \lambda, \theta=0^{\circ}$ for different central loads, $\mathrm{Z}_{\mathrm{L}}$.

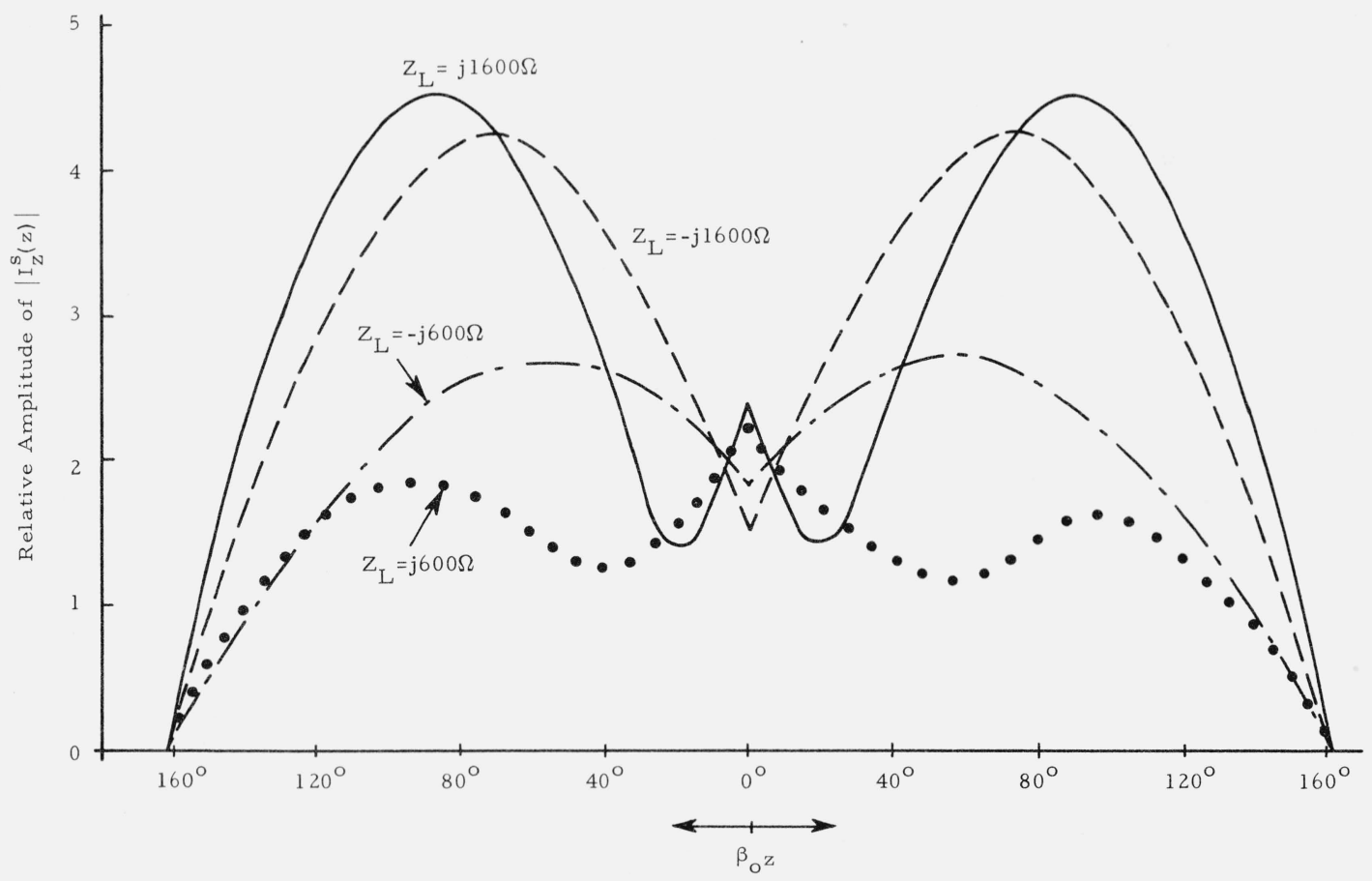

FIGURE 5. Current distribution, $\mathrm{I}_{z}^{\mathrm{s}}(\mathrm{z})$, on cylinder of $\mathrm{h}=0.45 \lambda, \mathrm{a}=0.0173 \lambda, \theta=0^{\circ}$ for different central loads, $\mathrm{Z}_{\mathrm{L}}$. 


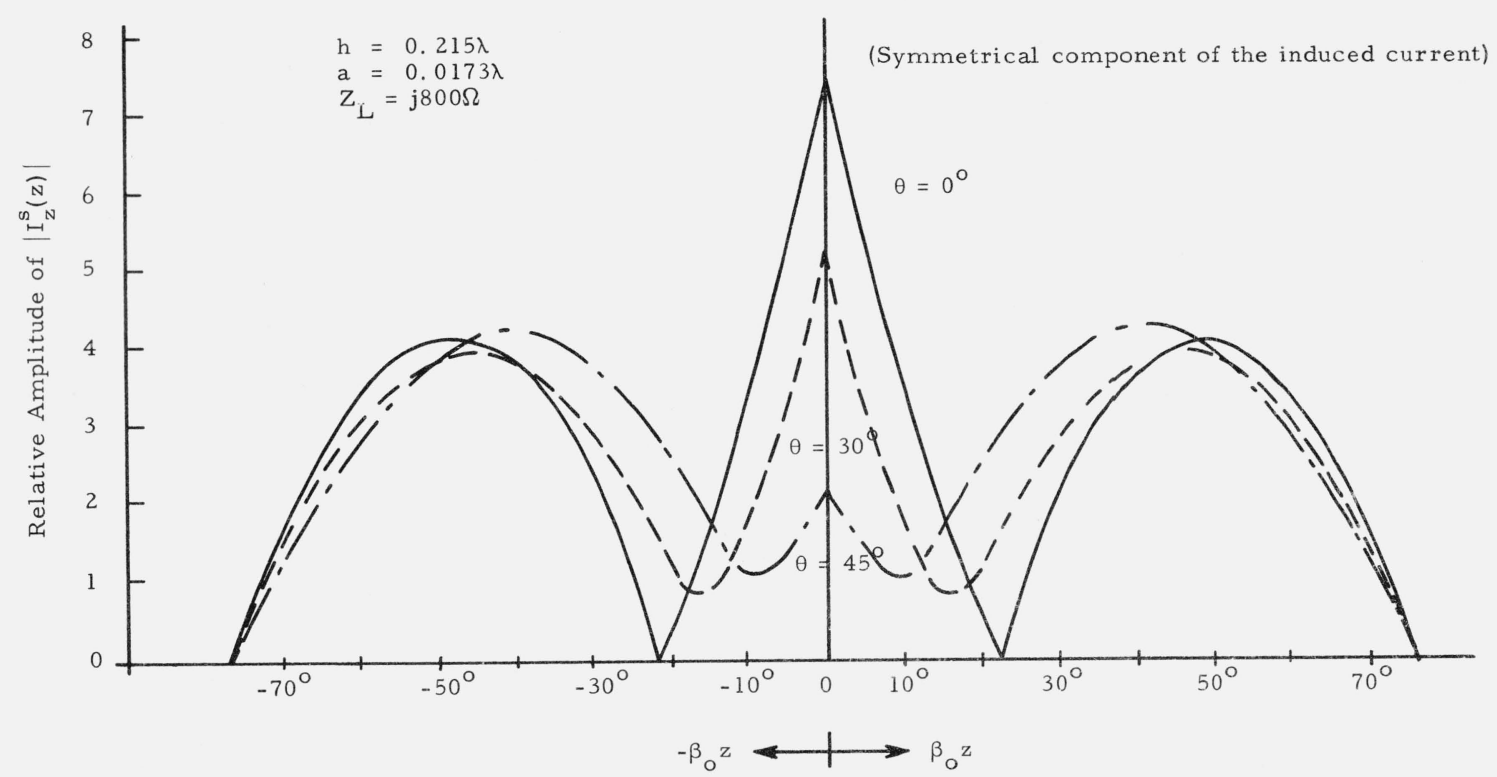

FIGURE 6. Distribution of $\mathrm{I}_{\mathrm{z}}^{\mathrm{s}}(\mathrm{z})$ along a resonant cylinder for different incident angles.

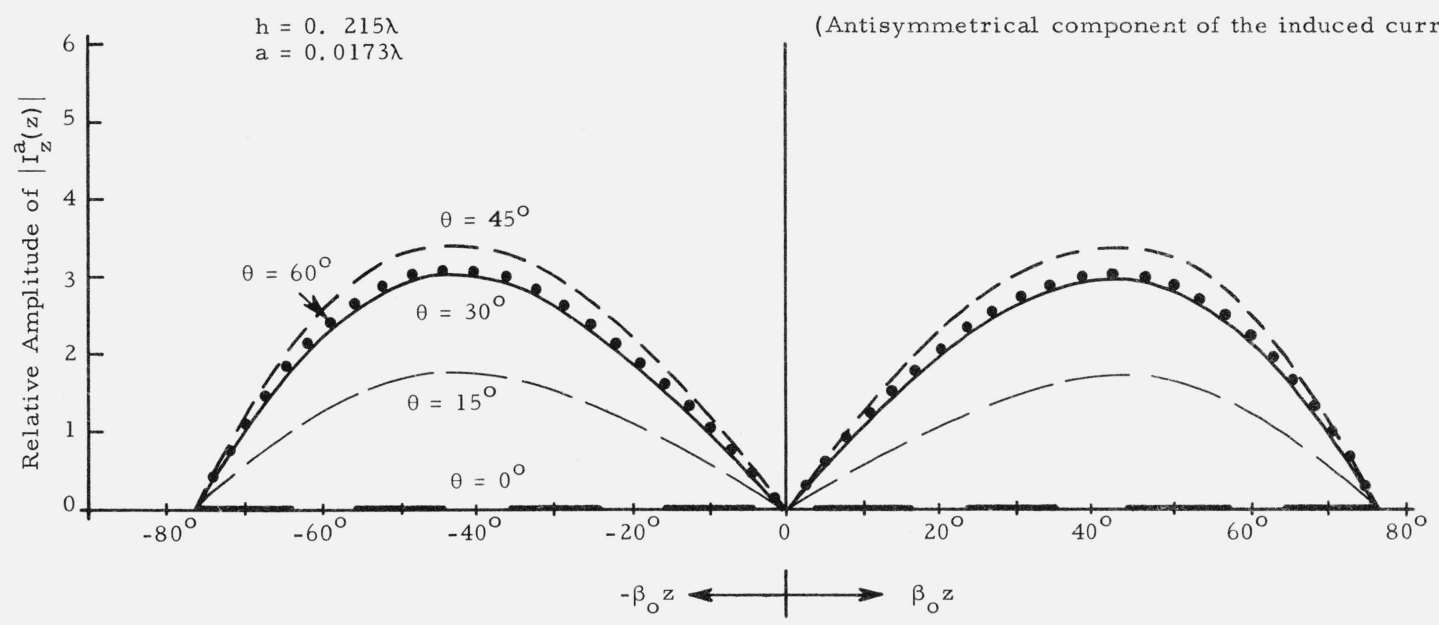

FIGURE 7. Distribution of $\mathrm{I}_{\mathrm{z}}^{\mathrm{a}}(\mathrm{z})$ along a resonant cylinder for different incident angles.

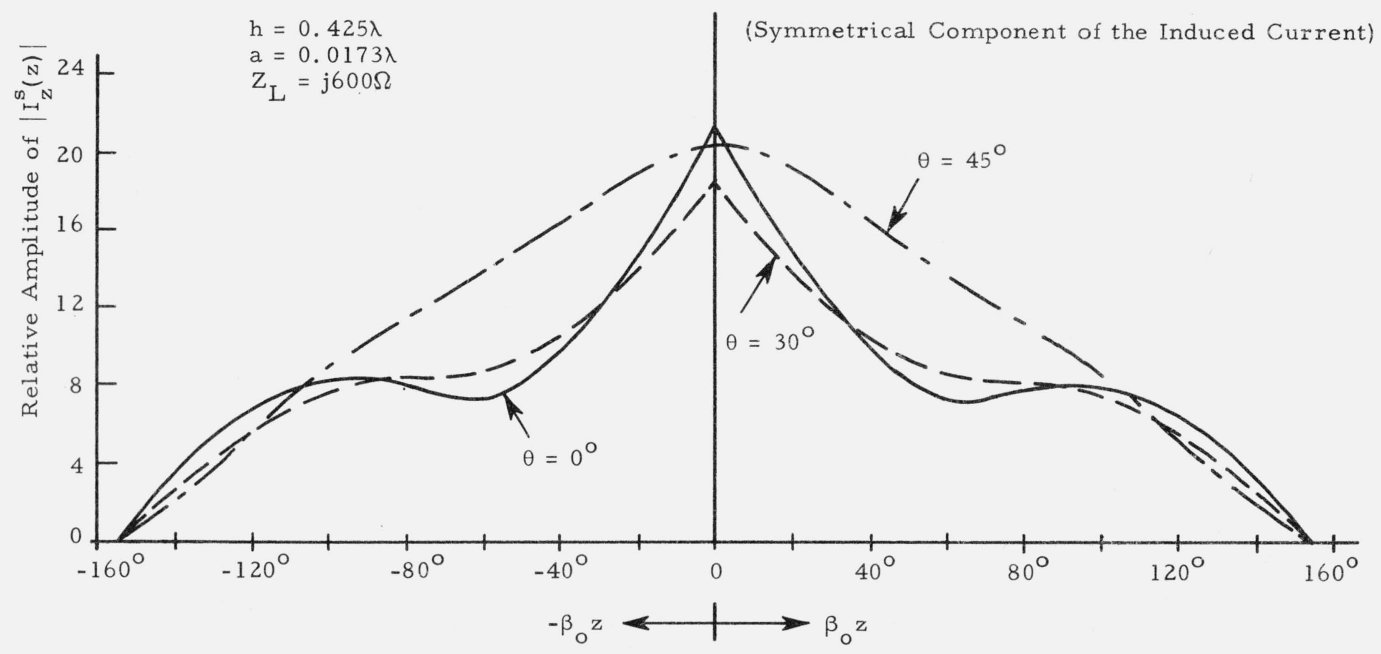

FIGURE 8. Distribution of $\mathrm{I}_{\mathrm{z}}^{\mathrm{s}}(\mathrm{z})$ along an antiresonant cylinder for different incident angles. 


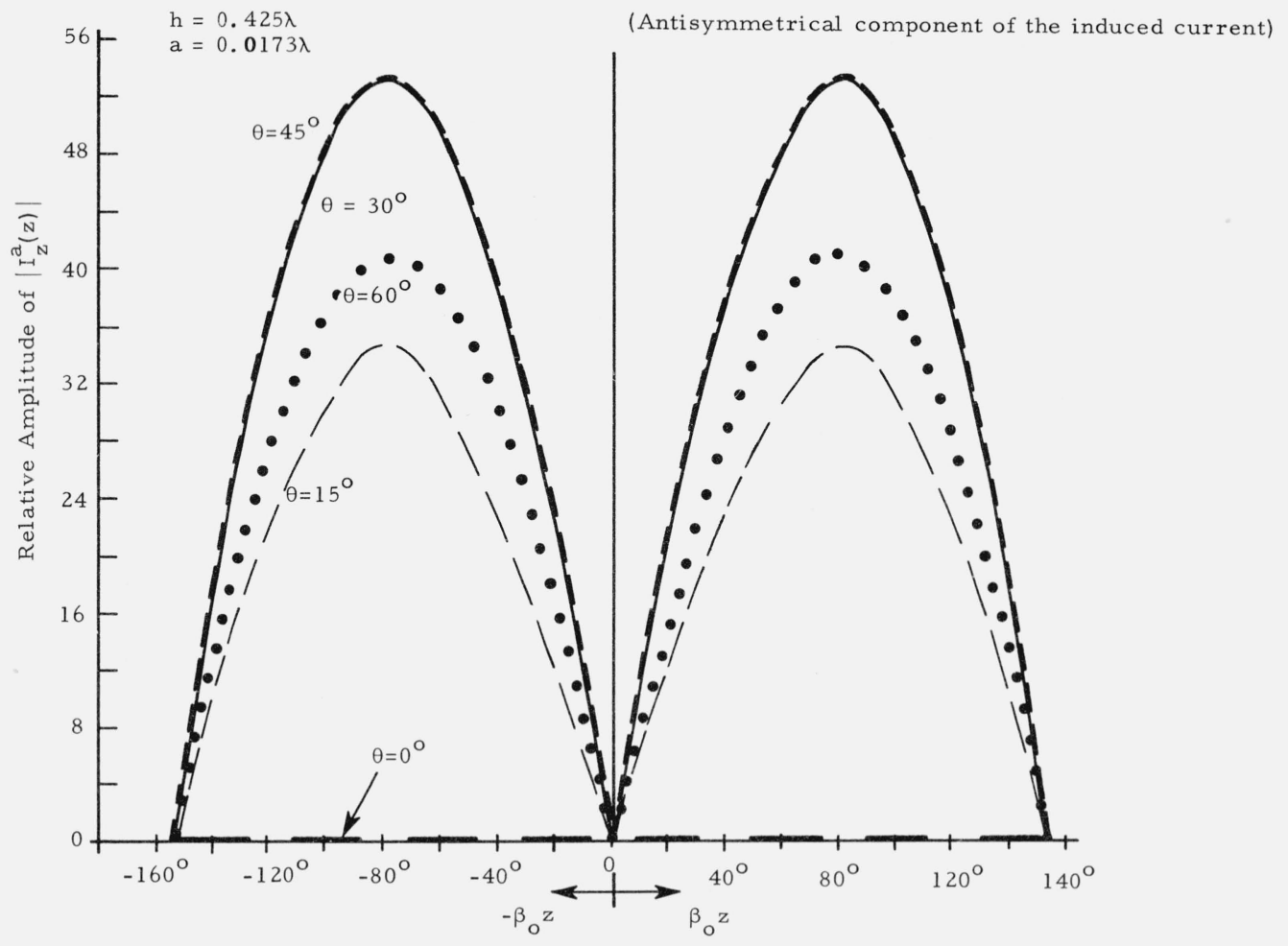

FIGURE 9. Distribution of $\mathrm{I}_{\mathbf{z}}^{\mathrm{a}}(\mathbf{z})$ along an antiresonant cylinder for different incident angles.

\section{Backscattering of a Center-Loaded Cylinder Illuminated by a Plane Wave at an Arbitrary Angle}

The induced current on a center-loaded cylinder illuminated by a plane wave at an arbitrary angle has been obtained in the preceding sections. Using the solution obtained there we can calculate the backscattered field.

\subsection{Backscattered Field of a Center-Loaded Cylinder}

With the geometry of figure 1 and the solution of the induced current as expressed in (17) and (22) we proceed as follows:

The symmetrical component of the induced current $I_{z}^{s}(z)$ maintains a vector potential at a point in the far zone of the cylinder in the direction of $\theta$ :

$$
A_{z}^{s}=\frac{\mu_{0}}{4 \pi} \frac{e^{-j \beta_{0} R_{0}}}{R_{0}} \int_{-h}^{h} I_{z}^{s}(z) e^{j \beta} 0^{z \sin \theta} d z
$$

where $I_{z}^{s}(z)$ can be obtained from (17) and where $R_{0}$ is the distance between the center of the cylinder and an observation point.

Performing the indicated integration in (24), the final expression for $A_{z}^{s}$ becomes

$$
\begin{aligned}
A_{z}^{s}(\theta)= & \frac{j \mu_{0} E_{0}}{120 \pi \beta_{0}^{2}} \frac{e^{-j \beta_{0} R_{0}}}{\mathrm{R}_{0}}\left\{\left[\frac{\cos \left(\beta_{0} h \sin \theta\right)-M_{2} T_{\theta a}-N_{2} T_{s a}}{\cos \beta_{0} h-M_{1} T_{c a}-N_{1} T_{s a}}\right] \frac{2 M_{1}}{\cos ^{3} \theta \sin \theta}\right. \\
\cdot\left[\sin \beta_{0} h \sin \theta \cos \left(\beta_{0} h \sin \theta\right)-\cos \beta_{0} h \sin \left(\beta_{0} h \sin \theta\right)\right] & \\
+ & -\left[\frac{N_{1} \cos \left(\beta_{0} h \sin \theta\right)-N_{1} M_{2} T_{\theta a}+M_{1} N_{2} T_{c a}-N_{2} \cos \beta_{0} h}{\cos \beta_{0} h-M_{1} T_{c a}-N_{1} T_{s a}}\right] \frac{2\left[\cos \left(\beta_{0} h \sin \theta\right)-\cos \beta_{0} h\right]}{\cos ^{3} \theta} \\
& \left.-\frac{M_{2}}{2 \sin \theta \cos \theta}\left[2 \beta_{0} h \sin \theta-\sin \left(2 \beta_{0} h \sin \theta\right)\right]\right\} .
\end{aligned}
$$


Similarly, the antisymmetrical component of the induced current $I_{z}^{a}(z)$ maintains a vector potential at a point in the far zone of the cylinder in the direction of $\theta$ :

$$
A_{z}^{a}=\frac{\mu_{0}}{4 \pi} \frac{e^{-j \beta_{0} R_{0}}}{R_{0}} \int_{-h}^{h} I_{z}^{a}(z) e^{j \beta_{0} z \sin \theta} d a
$$

where $I_{z}^{a}$ can be obtained from (22). After the integration in (26) is performed,

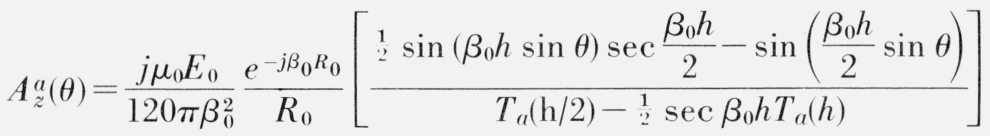

$$
\begin{aligned}
& \cdot \frac{1}{2 \cos ^{3} \theta \sin \theta}\left\{\sin \beta_{0} h\left[\left(1+\sin ^{2} \theta\right) \sin \left(2 \beta_{0} h \sin \theta\right)-2 \beta_{0} h \sin \theta \cos ^{2} \theta\right]\right. \\
& \left.-4 \cos \beta_{0} h \sin \theta \sin ^{2}\left(\beta_{0} h \sin \theta\right)\right\} .
\end{aligned}
$$

To obtain an expression for the backscattered field, the following argument is employed: The total vector potential maintained by the induced current on the cylinder is

$$
A_{z}(\theta)=A_{z}^{s}(\theta)+A_{z}^{a}(\theta) .
$$

The scattered electric field in the far zone of the cylinder due to the induced current is

$$
E_{\theta}=-j \omega A_{\theta}=j \omega A_{z} \cos \theta
$$

and the Poynting power density of the scattered field is

$$
P=\frac{1}{2 \zeta_{0}}\left|E_{\theta}\right|^{2} \text {. }
$$

Hence, using the values of $A_{z}^{s}(\theta)$ from $(25)$ and $A_{z}^{a}(\theta)$ from (27), the final expression for the backscattered field becomes

$$
\begin{aligned}
E_{\theta}(\theta)=-\frac{E_{0}}{\beta_{0}} \frac{e^{-j \beta_{0} R_{0}}}{R_{0}}\{ & {\left[\frac{\cos \left(\beta_{0} h \sin \theta\right)-M_{2} T_{\theta a}-N_{2} T_{s a}}{\cos \beta_{0} h-M_{1} T_{c a}-N_{1} T_{s a}}\right] \frac{2 M_{1}}{\cos ^{2} \theta \sin \theta} } \\
& \cdot\left[\sin \beta_{0} h \sin \theta \cos \left(\beta_{0} h \sin \theta\right)-\cos \beta_{0} h \sin \left(\beta_{0} h \sin \theta\right)\right] \\
& +\left[\frac{N_{1} \cos \left(\beta_{0} h \sin \theta\right)-N_{1} M_{2} T_{\theta a}+M_{1} N_{2} T_{c a}-N_{2} \cos \beta_{0} h}{\cos \beta_{0} h-M_{1} T_{c a}-N_{1} T_{s a}}\right] \\
& +\frac{2\left[\cos \left(\beta_{0} h \sin \theta\right)-\cos \beta_{0} h\right]}{\cos ^{2} \theta}-\frac{M_{2}}{2 \sin \theta}\left[2 \beta_{0} h \sin \theta-\sin \left(2 \beta_{0} h \sin \theta\right)\right] \\
& {\left[\frac{1}{\sin \left(\beta_{0} h \sin \theta\right) \sec \frac{\beta_{0} h}{2}-\sin \left(\frac{\beta_{0} h}{2}\right) \sin \theta}\right] \frac{1}{2 \cos ^{2} \theta \sin \theta} }
\end{aligned}
$$

$\cdot\left[\sin \beta_{0} h\left[\left(1+\sin ^{2} \theta\right) \sin \left(2 \beta_{0} h \sin \theta\right)-2 \beta_{0} h \sin \theta \cos ^{2} \theta\right]\right.$

$$
\left.\left.-4 \cos \beta_{0} h \sin \theta \sin ^{2}\left(\beta_{0} h \sin \theta\right)\right]\right\} .
$$

Equation (31) gives the complete expression for the backscattered electric field of a center-loaded cylinder when illuminated by a plane wave with an electric field $E_{0}$ at an angle $\theta$ with respect to 
the normal to the cylinder. The radar cross sections of some loaded cylinders are calculated from (31) as functions of aspect angle $\theta$ in section 4.3. The calculated results are in excellent agreement with the experimental results.

It is noted that the radar cross section is usually defined as

$$
\sigma=\lim _{R_{0} \rightarrow \infty} 4 \pi R_{0}^{2}\left|\frac{\mathrm{E}_{\theta}(\theta)}{E_{0}}\right|^{2} .
$$

\subsection{Optimum Loading for Zero Broadside Backscattering}

Before we show some numerical examples of the radar cross sections of loaded cylinders, we will consider an optimum loading to achieve zero backscattering in the broadside direction in this section.

When $E_{\phi}(\theta)$ in (31) is calculated as a function of $\theta$ for a resonant cylinder a large peak occurs at the broadside direction $\left(\theta=0^{\circ}\right)$. This peak is contributed by $I_{z}^{s}(z)$ on the cylinder and the contribution due to the small $I_{z}^{q}(z)$ in the off-broadside direction is insignificant in this case. When $E_{\theta}(\theta)$ is calculated for an antiresonant cylinder, there are three possible peaks. The first peak at $\theta=0^{\circ}$ is due to $I_{z}^{s}(z)$ and other peaks at the off-broadside directions are due to $I_{z}^{a}(z)$, which can be very large on an antiresonant cylinder. Since $I_{z}^{s}(z)$ can be changed greatly by a center load we seek an optimum loading which modifies $I_{z}^{s}(z)$ so that the backscattering in the broadside direction vanishes. The large backscattering in the off-broadside direction due to $I_{z}^{a}(z)$ is not considered here.

To make the broadside backscattering vanish, we can let

$$
\mathrm{E}_{\theta}\left(\theta=0^{\circ}\right)=0 .
$$

Since

$$
E_{\theta}\left(\theta=0^{\circ}\right)=-\frac{2 E_{0}}{\beta_{0}} \frac{e^{-j \beta_{0} R_{0}}}{R_{0}}\left[\frac{M_{1}\left(\sin \beta_{0} h-\beta_{0} h \cos \beta_{0} h\right)+N_{1}\left(1-\cos \beta_{0} h\right)}{\cos \beta_{0} h-M_{1} T_{c u}-N_{1} T_{s a}}\right],
$$

(33) implies

$$
\frac{N_{1}}{M_{1}}=-\frac{\sin \beta_{0} h-\beta_{0} h \cos \beta_{0} h}{1-\cos \beta_{0} h} .
$$

If (18) and (19) are substituted in (35), an optimum impedance $\left[Z_{L}\right]_{0}$ can be determined as

$$
\left[Z_{L}\right]_{0}=\frac{-j 60 T_{s d}\left(1-\beta_{0} h \cot \beta_{0} h\right)}{2 \cos \beta_{0} h-2+\beta_{0} h \sin \beta_{0} h}
$$

where $T_{s d}$ is defined in (48) in appendix 1.

This optimum impedance has been obtained by Chen and Liepa [1964a] and is included here for convenience in discussing some results in the next section. A numerical example is given in figure 10 in which the optimum central impedance $\left[Z_{L}\right]$, for zero broadside backscattering from a cylinder of radius $a=0.0173 \lambda$ is calculated as a function of the cylinder length. In figure 10 , we observe that, in general, the optimum loading for zero broadside backscattering requires an impedance with both reactive and resistive components. For a cylinder shorter than one wavelength a passive impedance is adequate but for a cylinder longer than one wavelength an active impedance which has a negative resistance is needed to reduce the broadside backscattering to zero. 


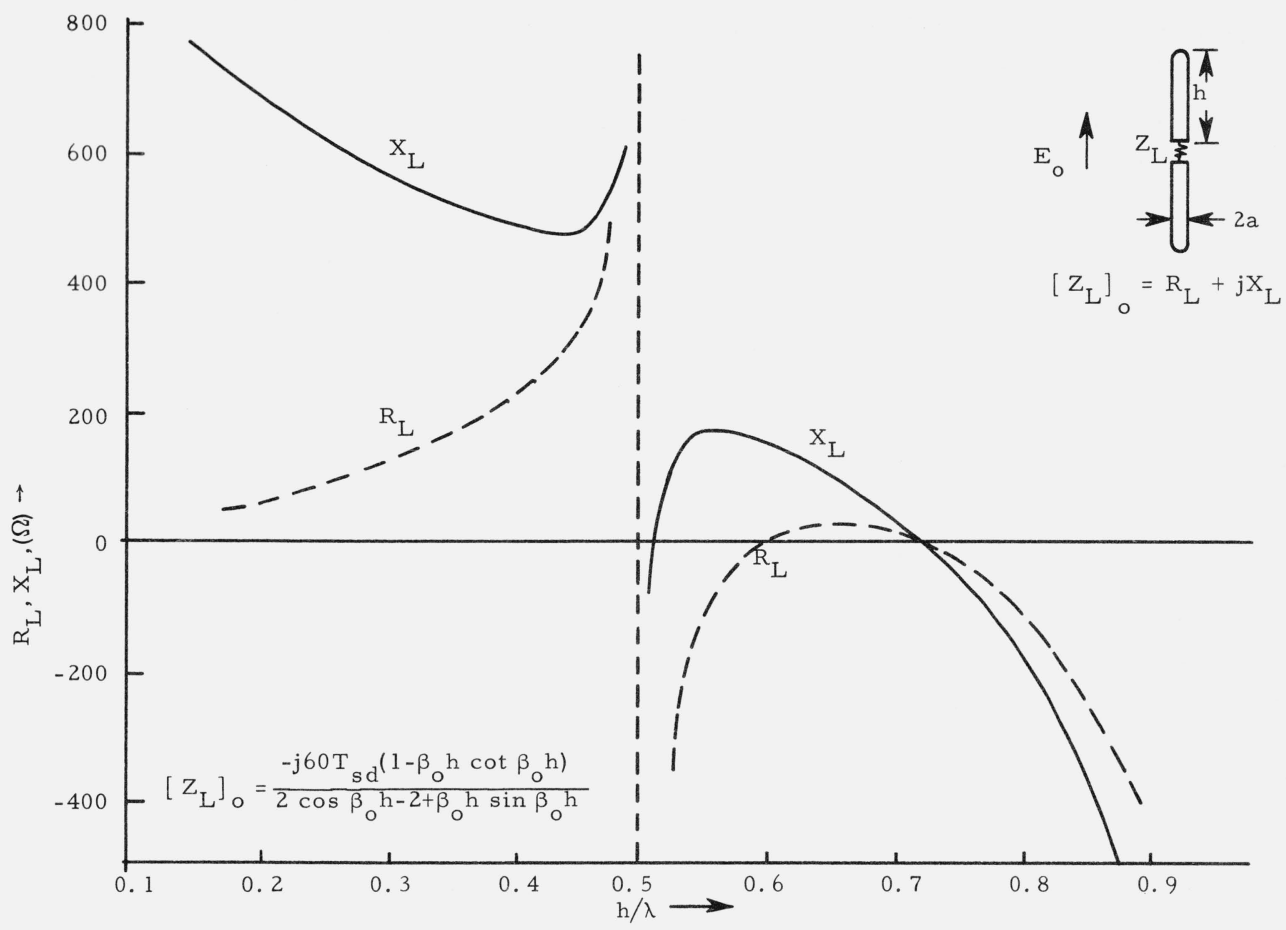

FIGURE 10. Optimum central impedance $\left[\mathrm{Z}_{\mathrm{L}}\right]_{0}$ for zero broadside backscattering from cylinder of radius a $=0.0173 \lambda$, as function of cylinder length.

\subsection{Numerical Examples on the Radar Cross Sections of Loaded Cylinders}

In this section the radar cross sections of a resonant and an antiresonant cylinder with various loadings are calculated. The theoretical results are compared with the experimental results by Chen and Liepa [1964b] and excellent agreement is obtained. Figure 11 shows the backscattering cross section of a resonant cylinder $(2 h=0.43 \lambda)$ as a function of the aspect angle for three different loadings. When $Z_{L}=0$ (a nonloaded cylinder) the cross section is very large. When the same cylinder is loaded with an infinite impedance $\left(Z_{L}=\infty\right)$, the backscattering cross section is reduced about $20 \mathrm{~dB}$. If the loading is adjusted close to the optimum value $\left(Z_{L}=j 600 \Omega\right)$, a reduction of more than $30 \mathrm{~dB}$ is obtained. The optimum loading $\left[Z_{L}\right]_{0}$ for this resonant cylinder is $65+j 626 \Omega$. The agreement between theory and experiment is excellent.

Figures 12 through 14 show the radar cross sections of an antiresonant cylinder $(2 h=0.85 \lambda)$ as a function of the aspect angle for three different loadings. When the cylinder is not loaded $\left(Z_{L}=0\right)$, the backscattering is approximately constant over the aspect angle range of $0<\theta<50^{\circ}$ with a slight maximum at $\theta=40^{\circ}$. The theoretical and the experimental results for this case are compared in figure 12 in which the zero- $\mathrm{dB}$ level is chosen to have the same absolute scale as in figure 11.

Figure 13 shows the theoretical curve for $Z_{L}=j 300 \Omega$ compared with an experimental curve for $Z_{L}=j 212 \Omega$. The point of interest is that for this loading the backscattering in the broadside direction is reduced considerably. These two curves, though with different loadings, agree quite well over most of the aspect range except for small $\theta$. The maximum backscattering occurs at $\theta=42^{\circ}$ and its amplitude is not reduced by the loading.

Figure 14 shows the theoretical curve for $Z_{L}=j 600 \Omega$ and a comparison with an experimental curve for $Z_{L}=j 415 \Omega$. The general behavior of these curves agrees very well. The maximum backscattering occurs at $\theta=42^{\circ}$ and, again, its amplitude is not reduced by loading. 


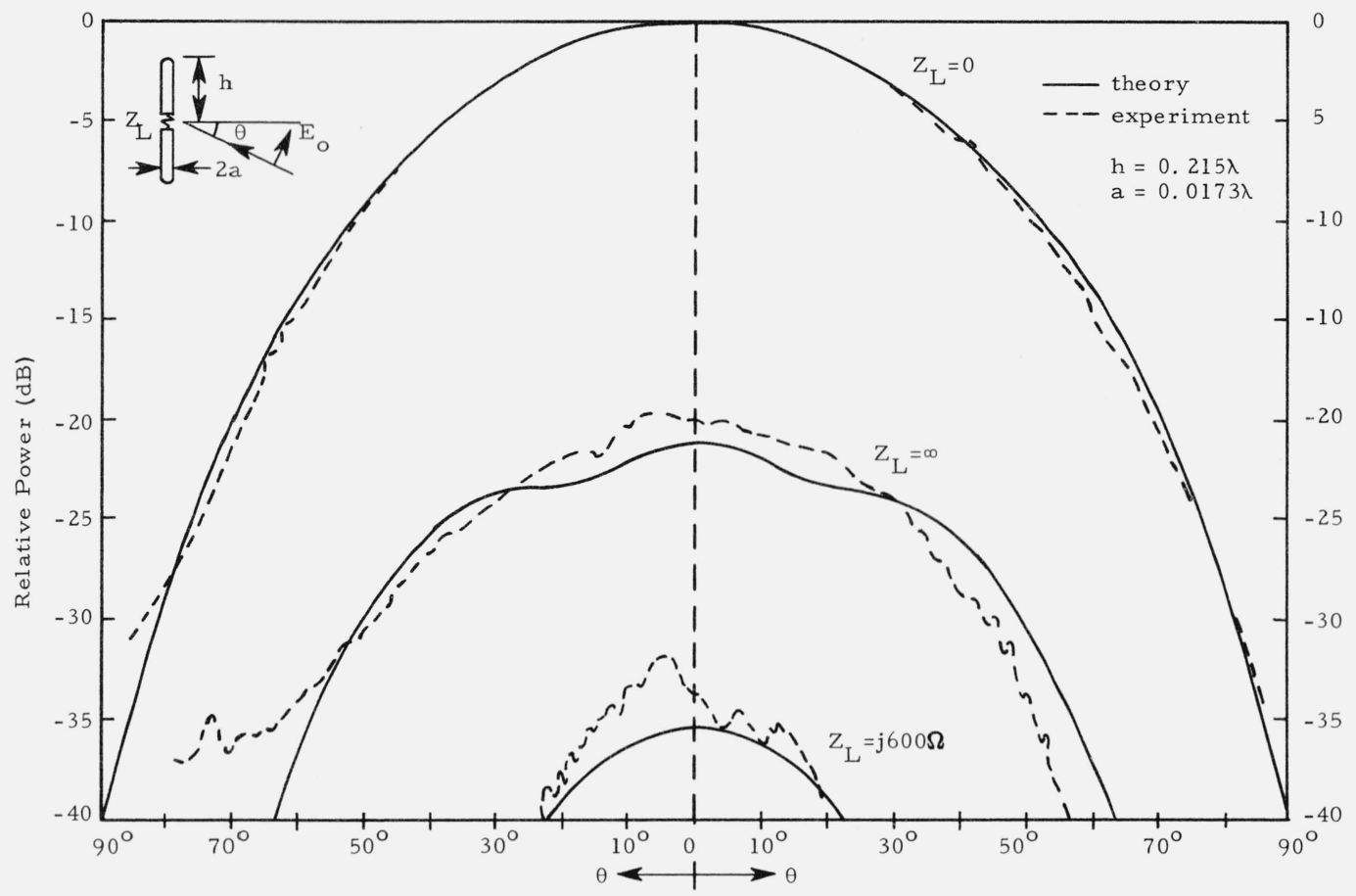

FIGURE 11. Backscattering cross sections of loaded cylinders versus aspect angle $(\theta)$.

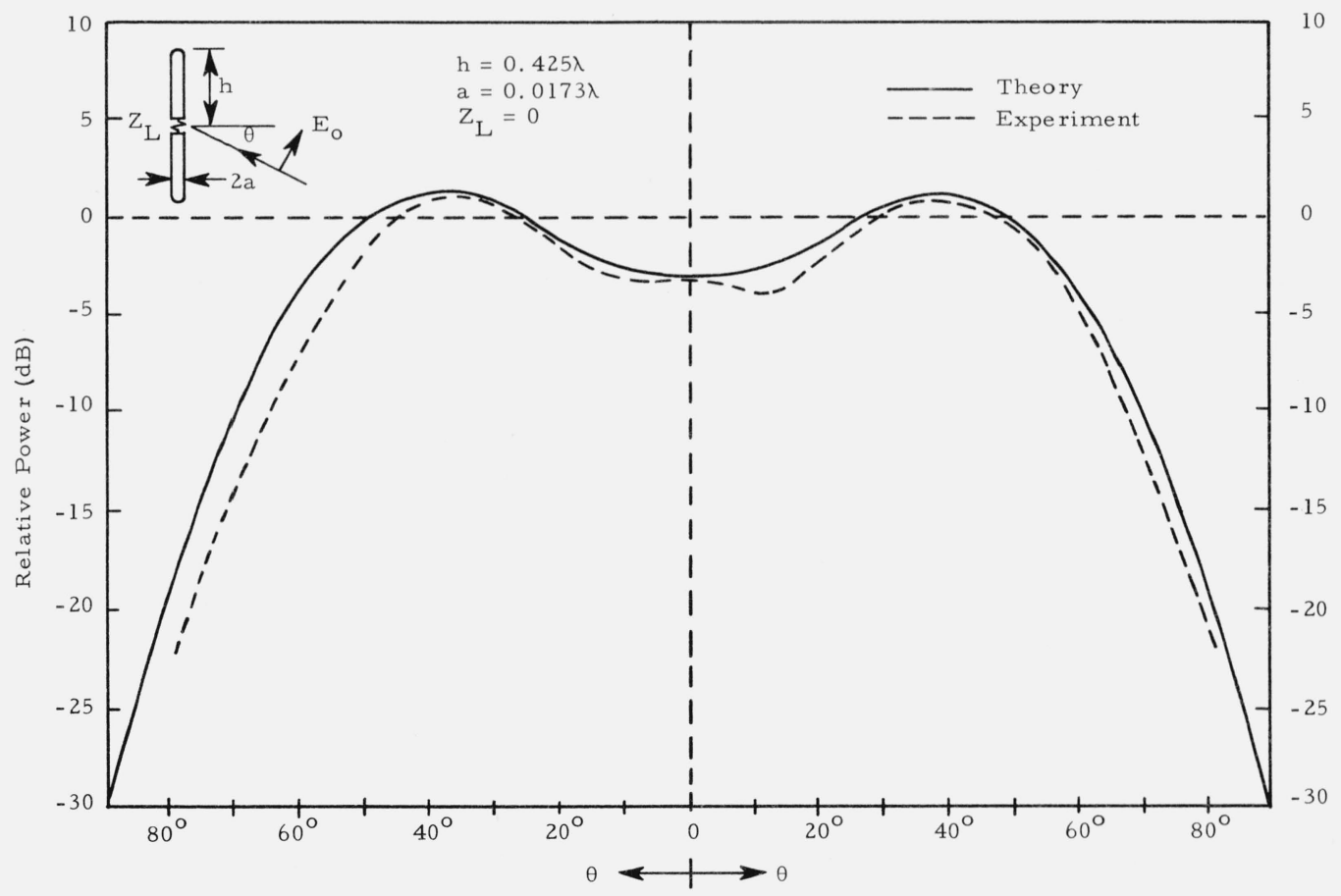

FIGURE 12. Backscattering cross section of loaded cylinder versus aspect angle $(\theta)$

It is noted that the optimum loading $\left[Z_{L}\right]_{0}$ for this antiresonant cylinder is about $230+j 435 \Omega$. In these three figures we find that the maximum cross section for an antiresonant cylinder at $\theta \simeq 40^{\circ}$ is not modified at all by a central loading. As mentioned before, this is due to the fact that this maximum backscattering is produced by the antisymmetrical component of the induced current, which is not affected by a central impedance. 


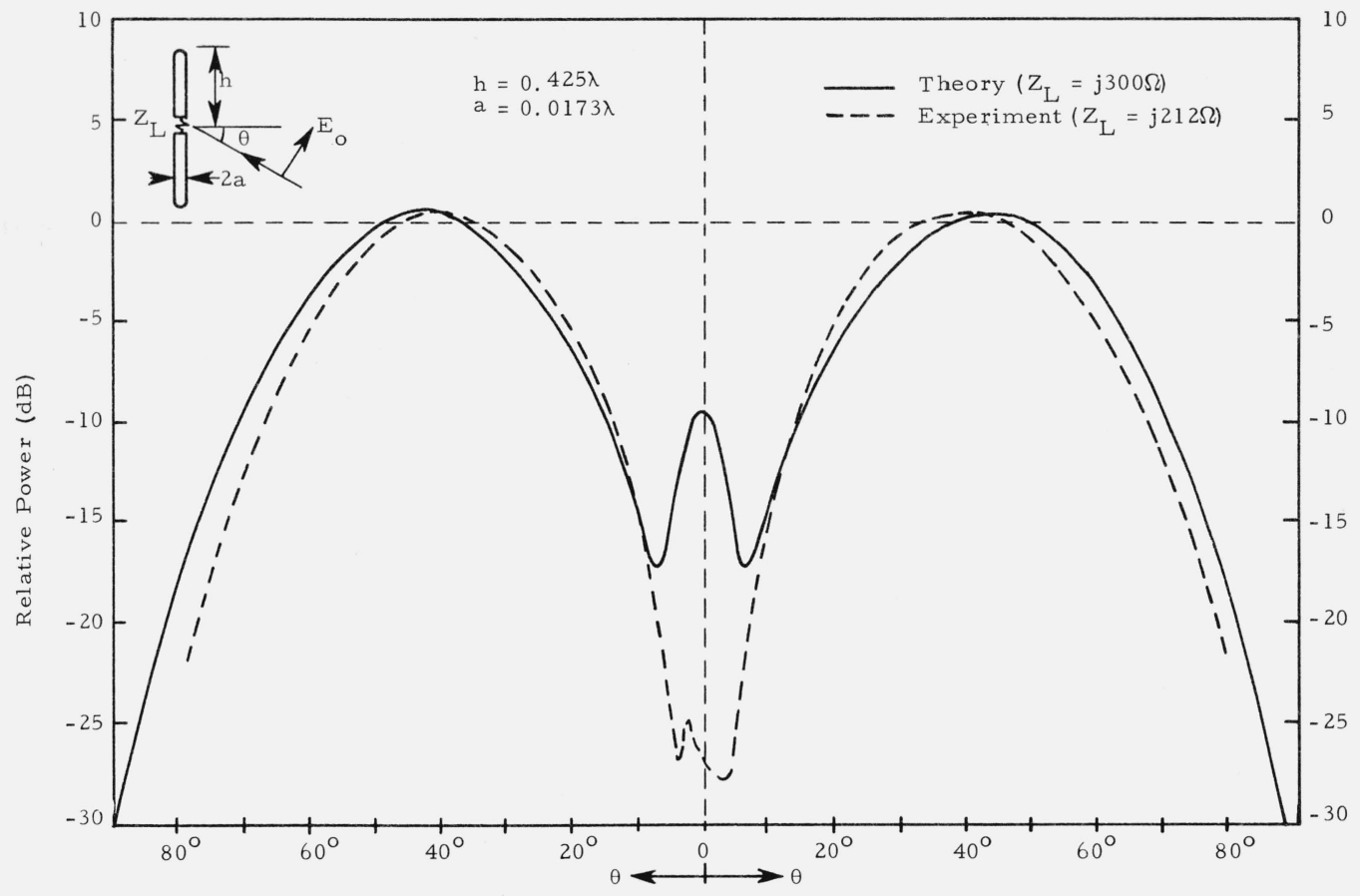

FIGURE 13. Backscattering cross section of loaded cylinder versus aspect angle $(\theta)$.

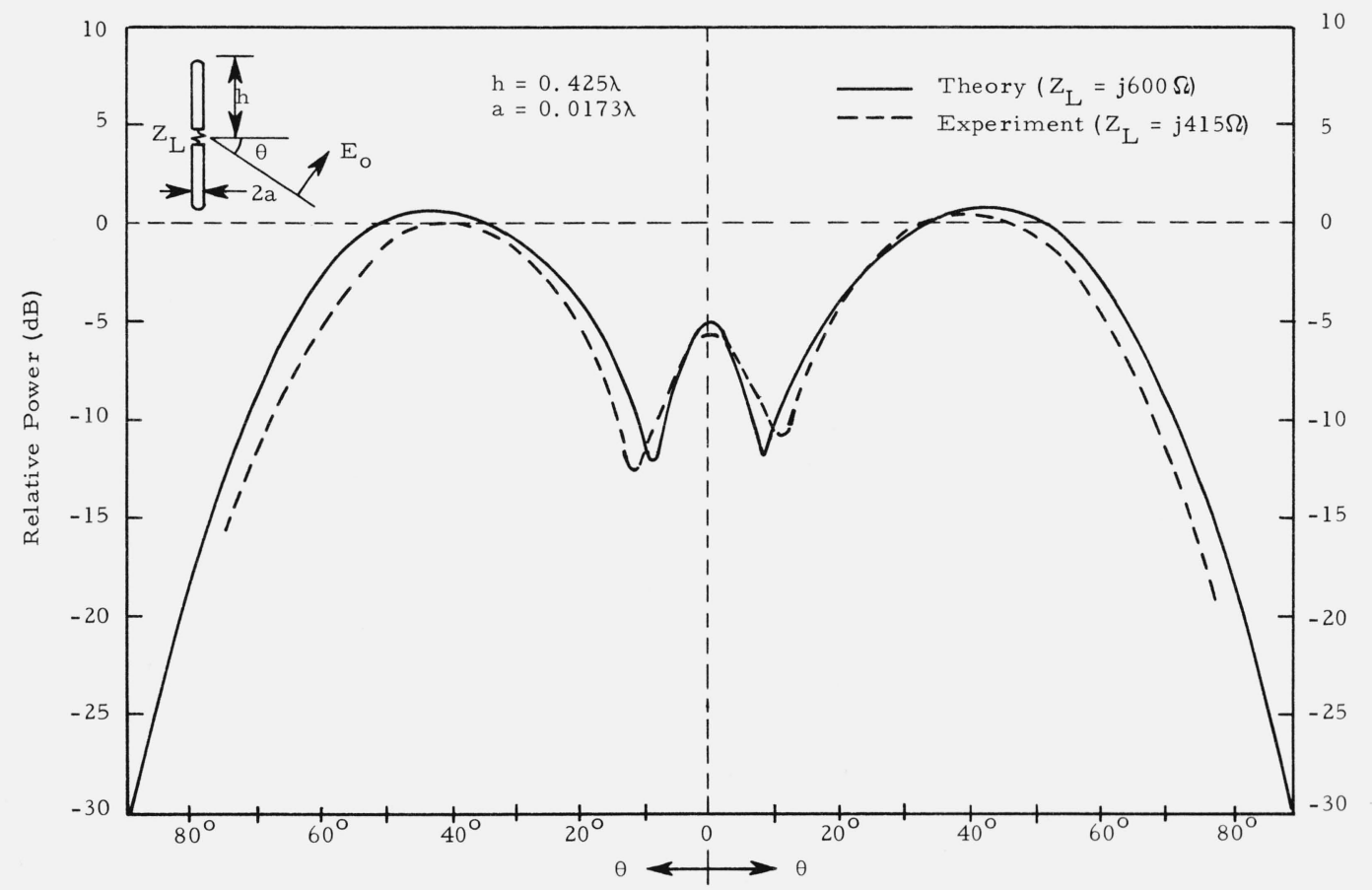

FIGURE 14. Backscattering cross section of loaded cylinder versus aspect angle $(\theta)$.

The excellent agreement between theory and experiment confirms the accuracy of the theory and also the feasibility of the reactive loading technique for the reduction of the radar cross section of a metallic body. 


\section{Conclusion}

In this paper we have shown the effectiveness of a loading technique for the reduction of the radar cross section of a metallic body. An optimum loading developed in (36) can be used to reduce the radar cross section of a resonant cylinder more than $30 \mathrm{~dB}$ in any aspect angle. For an antiresonant cylinder an optimum loading can be used to reduce the broadside cross section to an arbitrarily small value but it cannot modify the off-broadside cross section, which is due to the antisymmetrical component of the induced current. To reduce the radar cross section of an antiresonant cylinder over a wide aspect range, the double- or multiple-loading technique is suggested. The study on the double-loading technique will be published later.

The author is grateful to R. E. Hiatt, T. B. A. Senior, and B. Harrison for helpful suggestions, to V. Liepa and E. Knott for experimental measurements, and to H. Hunter and J. Ducmanis for their numerical calculations.

\section{Appendix 1. Determination of $I_{z}^{s}(z)$}

Instead of solving (14) directly for $I_{z}^{s}(z)$, for convenience we will start from (11), from which $c_{1}$ can be expressed as

$$
C_{1}=\sec \beta_{0} h\left[j v_{0} A_{z}^{s}(h)-\frac{E_{0}}{\beta_{0} \cos \theta} \cos \left(\beta_{0} h \sin \theta\right)+\frac{1}{2} Z_{L} I_{0} \sin \beta_{0} h\right]
$$

With (11) and (37), we obtain

$A_{z}(z)-A_{z}(h)$

$$
\begin{aligned}
& =\frac{-j}{v_{0}} \sec \beta_{0} h\left\{\left[j v_{0} A_{z}^{s}(h)-\frac{E_{0}}{\beta_{0} \cos \theta} \cos \left(\beta_{0} h \sin \theta\right)\right]\left(\cos \beta_{0} z-\cos \beta_{0} h\right)\right. \\
& \left.+\frac{1}{2} Z_{L} I_{0} \sin \beta_{0}(h-|z|)+\frac{E_{0} \cos \beta_{0} h}{\beta_{0} \cos \theta}\left[\cos \left(\beta_{0} z \sin \theta\right)-\cos \left(\beta_{0} h \sin \theta\right)\right]\right\}
\end{aligned}
$$

With the help of (14), another integral equation for $I_{z}^{s}(z)$ is obtained as follows:

$$
\begin{aligned}
& \int_{-h}^{h} I_{z}^{s}\left(s^{\prime}\right) K_{d}\left(z, z^{\prime}\right) d z^{\prime} \\
& =\frac{-j 4 \pi}{\zeta_{0}} \sec \beta_{0} h\left\{\left[j v_{0} A_{z}^{s}(h)-\frac{E_{0}}{\beta_{0} \cos \theta} \cos \left(\beta_{0} h \sin \theta\right)\right]\left(\cos \beta_{0} z-\cos \beta_{0} h\right)\right. \\
& \left.\quad+\frac{1}{2} Z_{L} I_{0} \sin \beta_{0}(h-|z|)+\frac{E_{0} \cos \beta_{0} h}{\beta_{0} \cos \theta}\left[\cos \left(\beta_{0} z \sin \theta\right)-\left(\beta_{0} h \sin \theta\right)\right]\right\}
\end{aligned}
$$

where

$$
K_{d}\left(z, z^{\prime}\right)=K_{a}\left(z, z^{\prime}\right)-K_{a}\left(h, z^{\prime}\right)
$$

and $\zeta_{0}$ is $120 \pi$. Equation (39) is valid for $-h<z<h$ but $A_{z}^{s}(h)$ and $I_{0}$ in the right-hand side of (39) are still unknown. 
However, due to the nature of kernel $K_{d}\left(z, z^{\prime}\right)$ which has a sharp peak at $z=z^{\prime}$, the right-hand side of (39) suggests a form for the solution of $I_{z}^{s}(z)$ as

$$
I_{z}^{s}(z)=C_{c}\left(\cos \beta_{0} z-\cos \beta_{0} h\right)+C_{s} \sin \beta_{0}(h-|z|)+C_{\theta}\left[\cos \left(\beta_{0} z \sin \theta\right)-\cos \left(\beta_{0} h \sin \theta\right)\right] .
$$

It is then reasonable to divide (39) into three parts as follows:

$C_{c} \int_{-h}^{h}\left(\cos \beta_{0} z^{\prime}-\cos \beta_{0} h\right) K_{d}\left(z, z^{\prime}\right) d z^{\prime}$

$$
\begin{array}{r}
=\frac{-j 4 \pi}{\zeta_{0}} \sec \beta_{0} h\left[j v_{0} A_{z}^{s}(h)-\frac{E_{0}}{\beta_{0} \cos \theta}\left(\beta_{0} h \sin \theta\right)\right]\left(\cos \beta_{0} z-\cos \beta_{0} h\right), \\
C_{s} \int_{-h}^{h} \sin \beta_{0}\left(h-\left|z^{\prime}\right|\right) K_{d}\left(z, z^{\prime}\right) d z^{\prime}=\frac{-j 2 \pi}{\zeta_{0}} \sec \beta_{0} h Z_{L} I_{0} \sin \beta_{0}(h-|z|),
\end{array}
$$

$C_{\theta} \int_{-h}^{h}\left[\cos \left(\beta_{0} z^{\prime} \sin \theta\right)-\cos \left(\beta_{0} h \sin \theta\right)\right] K_{d}\left(z, z^{\prime}\right) d z^{\prime}$

$$
=\frac{-j 4 \pi}{\zeta_{0}} \frac{E_{0}}{\beta_{0} \cos \theta}\left[\cos \left(\beta_{0} z \sin \theta\right)-\cos \left(\beta_{0} h \sin \theta\right)\right]
$$

Equations (42) through (44) are well matched at the end points, $z= \pm h$, where both sides of the equations become zero. Furthermore, the constants $C_{c}, C_{s}$, and $C_{\theta}$ can be determined by matching these equations at the center of the culinder, $z=0$. This matching yields

$$
C_{c}=\frac{-j 4 \pi}{\zeta_{0} T_{c d}} \sec \beta_{0} h\left[j v_{0} A_{z}^{s}(h)-\frac{E_{0}}{\beta_{0} \cos \theta} \cos \left(\beta_{0} h \sin \theta\right)\right]\left(1-\cos \beta_{0} h\right)
$$

where

$$
T_{c d}=\int_{-h}^{h}\left(\cos \beta_{0} z^{\prime}-\cos \beta_{0} h\right) K_{d}\left(0, z^{\prime}\right) d z^{\prime}
$$

and

$$
C_{s}=\frac{-j 2 \pi}{\zeta_{0} T_{s d}} \sec \beta_{0} h Z_{L} I_{0} \sin \beta_{0} h
$$

where

$$
T_{s d}=\int_{-h}^{h} \sin \beta_{0}\left(h-\left|z^{\prime}\right|\right) K_{d}\left(0, z^{\prime}\right) d z^{\prime}
$$

and

$$
C_{\theta}=\frac{-j 4 \pi}{\zeta_{0} T_{\theta d}} \frac{E_{0}}{\beta_{0} \cos \theta}\left[1-\cos \left(\beta_{0} h \sin \theta\right)\right]
$$

where

$$
T_{\theta d}=\int_{-h}^{h}\left[\cos \left(\beta_{0} z^{\prime} \sin \theta\right)-\cos \left(\beta_{0} h \sin \theta\right)\right] K_{d}\left(0, z^{\prime}\right) d z^{\prime} .
$$


The substitution of (45), (47), and (49) in (41) gives

$I_{z}^{s}(z)=\frac{-j 4 \pi}{\zeta_{0}}\left\{\frac{1}{T_{c d}}\left[j v_{0} A_{z}^{s}(h)-\frac{E_{0}}{\beta_{0} \cos \theta} \cos \left(\beta_{0} h \sin \theta\right)\right]\left(\sec \beta_{0} h-1\right)\left(\cos \beta_{0} z-\cos \beta_{0} h\right)\right.$

$$
\begin{gathered}
+\frac{1}{2 T_{s d}} Z_{L} I_{0} \tan \beta_{0} h \sin \beta_{0}(h-|z|) \\
\left.+\frac{1}{T_{\theta d}} \frac{E_{0}}{\beta_{0} \cos \theta}\left[1-\cos \left(\beta_{0} h \sin \theta\right)\right]\left[\cos \left(\beta_{0} z \sin \theta\right)-\cos \left(\beta_{0} h \sin \theta\right)\right]\right\} .
\end{gathered}
$$

In (51), $A_{z}^{s}(h)$ and $I_{0}$ are still unknown, but $I_{0}$ can be determined from (51).

By definition,

$$
I_{0} \equiv I_{z}(0)=I_{z}^{s}(0)+I_{z}^{a}(0), \text { but } I_{z}^{a}(0)=0
$$

hence

$$
I_{0}=I_{z}^{s}(0)
$$

Then $I_{0}$ can be expressed in terms of $A_{z}^{s}(h)$ by letting $z=0$ in (51), and after some algebraic manipulation (51) itself can be rearranged to give

$$
\begin{aligned}
I_{z}^{s}(z)=\frac{-j 4 \pi}{\zeta_{0}}\left\{\left[j v_{0} A_{z}^{s}(h)-\frac{E_{0}}{\beta_{0} \cos \theta} \cos \left(\beta_{0} h \sin \theta\right)\right]\right. \\
\cdot\left[M_{1}^{\prime}\left(\cos \beta_{0} z-\cos \beta_{0} h\right)+N_{1}^{\prime} \sin \beta_{0}(h-|z|)\right] \\
\left.+\frac{E_{0}}{\beta_{0} \cos \theta}\left[M_{2}\left[\cos \left(\beta_{0} z \sin \theta\right)-\cos \left(\beta_{0} h \sin \theta\right)\right]+N_{2} \sin \beta_{0}(h-|z|)\right]\right\}
\end{aligned}
$$

where

$$
\begin{aligned}
M_{1}^{\prime} & =\frac{1}{T_{c d}}\left(\sec \beta_{0} h-1\right) \\
N_{1}^{\prime} & =\frac{-Z_{L} \tan \beta_{0} h\left(\sec \beta_{0} h+\cos \beta_{0} h-2\right)}{T_{c d} Z_{L} \tan \beta_{0} h \sin \beta_{0} h-j 60 T_{c d} T_{s d}} \\
M_{2} & =\frac{1}{T_{\theta d}}\left[1-\cos \left(\beta_{0} h \sin \theta\right)\right] \\
N_{2} & =\frac{-Z_{L} \sin \beta_{0} h\left[1-\cos \left(\beta_{0} h \sin \theta\right)\right]^{2}}{T_{\theta d} Z_{L} \sin ^{2} \beta_{0} h-j 60 T_{\theta d} T_{s d} \cos \beta_{0} h}
\end{aligned}
$$

In (53) the remaining unknown is $A_{2}^{s}(h)$. To determine it we use the definition of the vector potential,

$$
A_{z}^{s}(h)=\frac{\mu_{0}}{4 \pi} \int_{-h}^{h} I_{z}^{s}\left(z^{\prime}\right) K_{a}\left(h, z^{\prime}\right) d z^{\prime}
$$

After substituting (53) in (58), $A_{z}^{s}(h)$ becomes

$$
A_{z}^{s}(h)=\frac{j E_{0}}{v_{0} \beta_{0} \cos \theta} \frac{\left[\cos \left(\beta_{0} h \sin \theta\right)\left(M_{1}^{\prime} T_{c a}+N_{1}^{\prime} T_{s a}\right)-\left(M_{2} T_{\theta a}+N_{2} T_{s a}\right)\right]}{1-M_{1}^{\prime} T_{c a}-N_{1}^{\prime} T_{s a}}
$$


where

$$
\begin{gathered}
T_{c a}=\int_{-h}^{h}\left(\cos \beta_{0} z^{\prime}-\cos \beta_{0} h\right) K_{a}\left(h, z^{\prime}\right) d z^{\prime} \\
T_{s a}=\int_{-h}^{h} \sin \beta_{0}\left(h-\left|z^{\prime}\right|\right) K_{a}\left(h, z^{\prime}\right) d z^{\prime} \\
T_{\theta a}=\int_{-h}^{h}\left[\cos \left(\beta_{0} z^{\prime} \sin \theta\right)-\cos \left(\beta_{0} h \sin \theta\right)\right] K_{a}\left(h, z^{\prime}\right) d z^{\prime} .
\end{gathered}
$$

If (59) is substituted in (53) and the result rearranged, the final form of the solution for $I_{z}^{s}(z)$ becomes

$$
\begin{array}{r}
I_{z}^{s}(z)=\frac{j E_{0}}{30 \beta_{0} \cos \theta}\left\{\left[\frac{\cos \left(\beta_{0} h \sin \theta\right)-M_{2} T_{\theta a}-N_{2} T_{s a}}{\cos \beta_{0} h-M_{1} T_{c a}-N_{1} T_{s a}}\right] M_{1}\left(\cos \beta_{0} z-\cos \beta_{0} h\right)\right. \\
+\left[\frac{N_{1} \cos \left(\beta_{0} h \sin \theta\right)-N_{1} M_{2} T_{\theta a}+M_{1} N_{2} T_{c a}-N_{2} \cos \beta_{0} h}{\cos \beta_{0} h-M_{1} T_{c a}-N_{1} T_{s a}}\right] \sin \beta_{0}(h-|z|) \\
\left.-M_{2}\left[\cos \left(\beta_{0} z \sin \theta\right)-\cos \left(\beta_{0} h \sin \theta\right)\right]\right\}
\end{array}
$$

where

$$
\begin{gathered}
M_{1}=\frac{1}{T_{c d}}\left(1-\cos \beta_{0} h\right) \\
N_{1}=\frac{-Z_{L} \sin \beta_{0} h\left(1-\cos \beta_{0} h\right)^{2}}{T_{c d} Z_{L} \sin ^{2} \beta_{0} h-j 60 T_{c d} T_{s d} \cos \beta_{0} h}
\end{gathered}
$$

and $M_{2}$ and $N_{2}$ are expressed in (56) and (57).

Equation (63) expresses $I_{z}^{S}(z)$ as a function of the cylinder size, the center load $Z_{L}$, and the incident angle $\theta$ of an EM wave.

As a matter of completeness and convenience, the integrals $T_{c d}, T_{s d}, T_{\theta d}, T_{c a}, T_{s a}$, and $T_{\theta a}$ are expressed in terms of better known integrals:

$$
\begin{aligned}
& T_{c d}=C_{a}(h, 0)-C_{a}(h, h)-\cos \beta_{0} h\left[E_{a}(h, 0)-E_{a}(h, h)\right] \\
& T_{s d}=\sin \beta_{0} h\left[C_{a}(h, 0)-C_{a}(h, h)\right]-\cos \beta_{0} h\left[S_{a}(h, 0)-S_{a}(h, h)\right] \\
& T_{\theta d}=C_{a}^{\theta}(h, 0)-C_{a}^{\theta}(h, h)-\cos \left(\beta_{0} h \sin \theta\right)\left[E_{a}(h, 0)-E_{a}(h, h)\right] \\
& T_{c a}=C_{a}(h, h)-\cos \beta_{0} h E_{a}(h, h) \\
& T_{s a}=\sin \beta_{0} h C_{a}(h, h)-\cos \beta_{0} h S_{a}(h, h) \\
& T_{\theta a}=C_{a}^{\theta}(h, h)-\cos \left(\beta_{0} h \sin \theta\right) E_{a}(h, h)
\end{aligned}
$$

where

$$
\begin{aligned}
& C_{a}(h, 0)=\int_{-, h}^{h} \cos \beta_{0} z^{\prime} K_{a}\left(0, z^{\prime}\right) d z^{\prime} \\
& C_{a}(h, h)=\int_{-h}^{h} \cos \beta_{0} z^{\prime} K_{a}\left(h, z^{\prime}\right) d z^{\prime}
\end{aligned}
$$




$$
\begin{aligned}
& E_{a}(h, 0)=\int_{-h}^{h} K_{a}\left(0, z^{\prime}\right) d z^{\prime} \\
& E_{a}(h, h)=\int_{-h}^{h} K_{a}\left(h, z^{\prime}\right) d z^{\prime} \\
& S_{a}(h, 0)=\int_{-h}^{h} \sin \beta_{0}\left|z^{\prime}\right| K_{a}\left(0, z^{\prime}\right) d z^{\prime} \\
& S_{a}(h, h)=\int_{-h}^{h} \sin \beta_{0}\left|z^{\prime}\right| K_{a}\left(h, z^{\prime}\right) d z^{\prime} \\
& C_{a}^{\theta}(h, 0)=\int_{-h}^{h} \cos \left(\beta_{0} z^{\prime} \sin \theta\right) K_{a}\left(0, z^{\prime}\right) d z^{\prime} \\
& C_{a}^{\theta}(h, h)=\int_{-h}^{h} \cos \left(\beta_{0} z^{\prime} \sin \theta\right) K_{a}\left(h, z^{\prime}\right) d z^{\prime} .
\end{aligned}
$$

The integrals of (72) through (79) can be calculated on a digital computer.

\section{Appendix 2. Determination of $I_{z}^{a}(z)$}

To determine $I_{z}^{a}(z)$, we start from (12). The constant $C_{2}$ can be expressed as

$$
C_{2}=\csc \beta_{0} h\left[j v_{0} A_{z}^{a}(h)+j \frac{E_{0}}{\beta_{0} \cos \theta} \sin \left(\beta_{0} h \sin \theta\right)\right]
$$

Substituting (80) in (15), we obtain

$$
\begin{aligned}
& \int_{-h}^{h} I_{z}^{a}\left(z^{\prime}\right) K_{a}\left(z, z^{\prime}\right) d z^{\prime} \\
& =\frac{4 \pi}{\zeta_{0}} \csc \beta_{0} h\left\{\frac{E_{0}}{\beta_{0} \cos \theta}\left[\sin \left(\beta_{0} h \sin \theta\right) \sin \beta_{0} z-\sin \beta_{0} h \sin \left(\beta_{0} z \sin \theta\right)\right]\right. \\
& \left.+v_{0} A_{z}^{a}(h) \sin \beta_{0} z\right\}
\end{aligned}
$$

If the solution for $I_{z}^{a}(z)$ is assumed to be

$$
I_{z}^{a}(z)=C_{a}\left[\sin \left(\beta_{0} h \sin \theta\right) \sin \beta_{0} z-\sin \beta_{0} h \sin \left(\beta_{0} z \sin \theta\right)\right],
$$

(81) is matched at $z=0$. We will match (81) at two more points.

If we set $z=h / 2$ in (81) and use the substitution of (82), the constant $C_{a}$ can be expressed as

$$
C_{a}=\frac{1}{T_{a}(h / 2)}\left\{\frac{2 \pi E_{0}}{\zeta_{0} \beta_{0} \cos \theta}\left[\sin \left(\beta_{0} h \sin \theta\right) \sec \frac{\beta_{0} h}{2}-2 \sin \left(\frac{\beta_{0} h}{2} \sin \theta\right)\right]+\frac{2 \pi}{\mu_{0}} \sec \frac{\beta_{0} h}{2} A_{z}^{a}(h)\right\}
$$

where

$$
T_{a}(h / 2)=\int_{-h}^{h}\left[\sin \left(\beta_{0} h \sin \theta\right) \sin \beta_{0} z^{\prime}-\sin \beta_{0} h \sin \left(\beta_{0} z^{\prime} \sin \theta\right)\right] K_{a}\left(\mathrm{~h} / 2, z^{\prime}\right) d z^{\prime} .
$$

By definition, $A_{z}^{a}(h)$ is

$$
A_{z}^{a}(h)=\frac{\mu_{0}}{4 \pi} \int_{-h}^{h} I_{z}^{a}\left(z^{\prime}\right) K_{a}\left(h, z^{\prime}\right) d z^{\prime}=\frac{\mu_{0}}{4 \pi} C_{a} T_{a}(h)
$$


where

$$
T_{a}(h)=\int_{-h}^{h}\left[\sin \left(\beta_{0} h \sin \theta\right) \sin \beta_{0} z^{\prime}-\sin \beta_{0} h \sin \left(\beta_{0} z^{\prime} \sin \theta\right)\right] K_{a}\left(h, z^{\prime}\right) d z^{\prime}
$$

From (83) and (85), $A_{z}^{a}(h)$ is determined as

$$
A_{z}^{a}(h)=\frac{E_{0} T_{a}(h)\left[\sin \left(\beta_{0} h \sin \theta\right) \sec \frac{\beta_{0} h}{2}-2 \sin \left(\frac{\beta_{0} h}{2} \sin \theta\right)\right]}{\omega \cos \theta\left[2 T_{a}(h / 2)-\sec \frac{\beta_{0} h}{2} T_{a}(h)\right]} .
$$

After substituting (83) and (87) into (82) we obtain the final form of the solution for $I_{z}^{a}(z)$,

$I_{z}^{a}(z)=\frac{E_{0}}{30 \beta_{0} \cos \theta}\left[\frac{! \sin \left(\beta_{0} h \sin \theta\right) \sec \frac{\beta_{0} h}{2}-\sin \left(\frac{\beta_{0} h}{2} \sin \theta\right)}{T_{a l}(h / 2)-\frac{1}{2} \sec \beta_{0} h T_{a l}(h)}\right]$

$$
\cdot\left[\sin \left(\beta_{0} h \sin \theta\right) \sin \beta_{0} z-\sin \beta_{0} h \sin \left(\beta_{0} z \sin \theta\right)\right] .
$$

Equation (88) gives the complete solution for the antisymmetrical component of the induced current on a cylinder. It is noted that $I_{z}^{a}(z)$ is a function of the cylinder size and the incidence angle $\theta$ only, and is entirely independent of the center load $Z_{L}$.

For convenience the integrals $T_{a}(h / 2)$ and $T_{a}(h)$ are expressed alternatively as follows:

$$
\begin{gathered}
T_{a}(h / 2)=\sin \left(\beta_{0} h \sin \theta\right) S_{{ }^{\prime \prime}}^{90}(h, h / 2)-\sin \beta_{0} h S_{\prime \prime}^{\theta}(h, h / 2) \\
T_{a}(h)=\sin \left(\beta_{0} h \sin \theta\right) S_{! \prime}^{90}(h, h)-\sin \beta_{0} h S_{l l}^{\theta}(h, h)
\end{gathered}
$$

where

$$
\begin{aligned}
S_{a}^{\theta}(h, h / 2) & =\int_{-h}^{h} \sin \left(\beta_{0} z^{\prime} \sin \theta\right) K_{a}\left(h / 2, z^{\prime}\right) d z^{\prime} \\
S_{a}^{\theta}(h, h) & =\int_{-h}^{h} \sin \left(\beta_{0} z^{\prime} \sin \theta\right) K_{a}\left(h, z^{\prime}\right) d z^{\prime} \\
S_{a}^{90}(h, h / 2) & =\int_{-h}^{h} \sin \beta_{0} z^{\prime} K_{a}\left(h / 2, z^{\prime}\right) d z^{\prime} \\
S_{l}^{90}(h, h) & =\int_{-h}^{h} \sin \beta_{0} z^{\prime} K_{a}\left(h, z^{\prime}\right) d z^{\prime} .
\end{aligned}
$$

The integrals in (91) through (94) can be readily calculated on a computer.

\section{References}

Ås, B. O., and H. J. Schmitt (1958), Backscattering cross section of reactively loaded cylindrical antennas, Harvard University Cruft Laboratory Scientific Report No. 18.

Chen, Kun-Mu, and V. Liepa (1964a), The minimization of the backscattering of a cylinder by central loading, IEEE Trans.

Ant. Prop. AP-12, No. 5, 576-582.

Chen, Kun-Mu, and V. Liepa (April 1964b), The minimization of the radar cross section of a cylinder by central loading,

University of Michigan Radiation Laboratory Scientific Report No. 5548-1-T.

Hu, Yueh-Ying (1958), Backscattering cross section of a center-loaded cylinder, IRE Trans. Ant. Prop. AP-6, 140-148. Iams, H. A. (1950), Radio wave conducting device, U.S. Patent No. 2,528,367.

King, R. W. P. (1956), The Theory of Linear Antennas, pp. 506-511 (Harvard University Press, Cambridge, Mass.).

Sletten, C. J. (1962), Air Force Cambridge Research Laboratories, private communication.

(Paper 69D11-581) 